\title{
VLBA observations of SiO masers towards Mira variable stars
}

\author{
W. D. Cotton ${ }^{1}$, B. Mennesson ${ }^{2}$, P. J. Diamond ${ }^{3}$, G. Perrin 4 , V. Coudé du Foresto ${ }^{4}$, G. Chagnon ${ }^{4}$, \\ H. J. van Langevelde ${ }^{5}$, S. Ridgway ${ }^{6}$, R. Waters ${ }^{7}$, W. Vlemmings ${ }^{8}$, S. Morel $^{9}$, \\ W. Traub ${ }^{9}$, N. Carleton ${ }^{9}$, and M. Lacasse ${ }^{9}$
}

\author{
1 National Radio Astronomy Observatory ${ }^{\star}, 520$ Edgemont Road, Charlottesville, VA 22903-2475, USA \\ 2 Jet Propulsion Lab., Interferometry Systems and Technology Section, California Institute of Technology, \\ 480 Oak Grove Drive, Pasadena, CA 91109, USA \\ 3 Jodrell Bank Observatory, University of Manchester, Macclesfield Cheshire, SK11 9DL, UK \\ 4 DESPA, Observatoire de Paris, section de Meudon, 5 place Jules Janssen, 92190 Meudon, France \\ 5 Joint Institute for VLBI in Europe, Postbus 2, 7990 AA Dwingeloo, The Netherlands \\ ${ }^{6}$ NOAO, 950 N. Cherry Ave., PO Box 26732, Tucson, AZ. 85726, USA \\ 7 Astronomical Institute, University of Amsterdam, Kruislaan 403, 1098 SJ Amsterdam, The Netherlands \\ 8 Leiden Observatory, PO Box 9513, 2300 RA Leiden, The Netherlands ${ }^{\star \star}$ \\ 9 Harvard-Smithsonian Center for Astrophysics, Cambridge, MA 02138, USA
}

Received 2 April 2003 / Accepted 10 October 2003

\begin{abstract}
We present new total intensity and linear polarization VLBA observations of the $v=2$ and $v=1 J=1-0$ maser transitions of $\mathrm{SiO}$ at 42.8 and $43.1 \mathrm{GHz}$ in a number of Mira variable stars over a substantial fraction of their pulsation periods. These observations were part of an observing program that also includes interferometric measurements at 2.2 and 3.6 micron (Mennesson et al. 2002); comparison of the results from different wavelengths allows studying the envelope independently of the poorly known distances to these stars. Nine stars were observed at from one to four epochs during 2001. The SiO emission is largely confined to rings which are smaller than the inner radius of the dust shells reported by Danchi et al. (1994). Two stars (U Orionis, R Aquarii) have maser rings with diameters corresponding to the size of the hot molecular layer as measured at 3.6 micron; in the other cases, the $\mathrm{SiO}$ rings are substantially larger. Variations of ring diameter for most, but not all stars, had an rms amplitude in agreement with the models of Humphreys et al. (2002) although the expected relationship between the diameter and pulsation phase was not seen. The ring diameter in U Orionis shows remarkably small variation. A correlation between the $2.2 / 3.6 \mu \mathrm{m}$ diameter ratio with that of the $\mathrm{SiO} / 3.6 \mu \mathrm{m}$ diameter ratio is likely due to differences in the opacities at 2.2 and $3.6 \mu \mathrm{m}$ in a molecular layer. A further correlation with the inner size of the dust shell reported by Danchi et al. (1994) suggest some differences in the temperature structure. Clear evidence is seen in R Aquarii for an equatorial disk similar to that reported by Hollis et al. (2001); rotation is possibly also detected in S Coronae Boralis.
\end{abstract}

Key words. stars: atmospheres - stars: AGB and post-AGB - stars: variables - radio lines: stars, Masers

\section{Introduction}

Mira variables are stars of a few solar masses which have reached the end of their lives and have moved to the Asymptotic Giant Branch (AGB) where the inner core of the star has collapsed and the outer envelope has become very extended and is in the process of losing its mass to the interstellar medium. Miras are pulsationally unstable and show regular brightness variations of many magnitudes in the visual. Since the envelopes of these stars are relatively cool, molecules form. Shocks in the inner stellar atmosphere (Humphreys et al. 2002)

Send offprint requests to: W. D. Cotton, e-mail: bcotton@nrao. edu

* The National Radio Astronomy Observatory (NRAO) is operated by Associated Universities Inc., under cooperative agreement with the National Science Foundation.

$\star \star$ Current address: Wouter Vlemmings, 524 Space Sciences Building, Cornell University, Ithaca, NY 14853-6801, USA. are thought to drive the molecular material to distances from the photosphere at which it can condense into dust; the radiation pressure on the dust then drives the stellar wind, causing substantial mass loss from the star. After the mass loss phase, Mira stars evolve into planetary nebulae.

The extended envelopes of many Miras and other long period variables exhibit molecular masers, especially in $\mathrm{OH}, \mathrm{H}_{2} \mathrm{O}$ and $\mathrm{SiO}$ in order of decreasing distance from the photosphere (Reid \& Moran 1981). The SiO masers occur within a few stellar radii of the stellar surface between the hot molecular inner envelope and the cooler region at $3-5 R_{\star}$ where the (silicate) dust forms; see Reid \& Menten (1997), Danchi et al. (1994). VLBI observations of the $\mathrm{SiO}$ masers in this dynamically complex region provide a powerful probe.

Indeed, the frequent observations of Diamond \& Kemball (1999) of TX Cam provide a wealth of information about the 
dynamics at the base of the stellar wind. Prior to these observations, the picture that had emerged of the evolution of the circumstellar envelopes around pulsating stars was based principally on IR spectroscopy of TiO by Hinkle et al. (1982) and the theoretical atmospheric piston models of Bowen (1988). This was supported by the early VLBA monitoring of $\mathrm{SiO}$ around R Aquarii (Boboltz et al. 1997) and additional theoretical work by Gray et al. (1998). However, the continuing VLBA observations of TX Cam generate images that do not follow this model at all; there is little evidence of global contraction of the envelope as observed in R Aquarii (Boboltz et al. 1997). Instead, for TX Cam, Diamond \& Kemball observe expansion predominantly although, other complex motions are evident.

Circumstellar $\mathrm{SiO}$ masers tend to occur in clumpy, partial rings centered on the central star (Diamond et al. 1994). (This is presumed to be due to the longer paths through constant velocity gas needed for the masers to develop.) Thus, these masers become markers for the layer in which the physical conditions necessary for the masers exist. The resolution available to VLBI observations is a very small fraction of the diameter of the ring; the limit on the accuracy of the measurement of the ring diameter is determined by the number and distribution of the maser spots. Much of the emission is confined in localized "spots" with lifetimes of a few months. The theory of maser emission is given in Elitzur (1991), Nedoluha \& Watson (1994), Elitzur (1993, 1996, 1998). Modeling of the dynamics of circumstellar masers is developed in Humphreys et al. (1996, 2002) which give plausible agreement with the observations.

Joint observations of the Mira S Ori with the ESO VLTI interferometer at $2.2 \mu \mathrm{m}$ and VLBA observations of $\mathrm{SiO}$ masers are reported by Wittkowski \& Bobolz (2003). This study has only a single epoch $\mathrm{SiO}$ measurement of S Ori but multiple observations at $2.2 \mu \mathrm{m}$ which showed the expected variation of the stellar diameter.

This paper reports on the results of VLBI monitoring of the $\mathrm{SiO}$ masers in a number of stars which are part of a joint radio/IR program with the FLUOR/TISIS interferometer at wavelengths of $2.2 \mu \mathrm{m}$ (Coude Du Foresto et al. 1998) and $3.6 \mu \mathrm{m}$ (Mennesson et al. 1999) on the SAO IOTA array (Traub et al. 2000) on Mt. Hopkins Arizona, USA. Sources were selected from the list of Engels \& Heske (1989) which were also observable with the FLUOR/IOTA interferometer.

The 2.2 and $3.6 \mu \mathrm{m}$ results have been reported by Mennesson et al. (2002) which show a large increase in apparent angular diameter from 2.2. to $3.6 \mu \mathrm{m}$. Narrow band observations carried out with IOTA/FLUOR in the continuum and the $\mathrm{H} 2 \mathrm{O}$ and $\mathrm{CO}$ bands in the $2.2 \mu \mathrm{m}$ atmospheric window and coupled with $3.6 \mu \mathrm{m}$ band observations made with IOTA/TISIS have lead to the development of a layer model in which the optical depth is wavelength dependent Perrin et al. (2003). Optical depths are found to vary between modecular and continuum bands and between the 2.2 and $3.6 \mu \mathrm{m}$ bands. The differential size between bands is therefore a combination of the contrast ratio between the modecular layer and the photosphere which is a function of temperature and of the wavelength dependence of optical depths. In the previous discussion of this model in Mennesson et al. (2002). the optical depths were set equal between the 2.2 and $3.6 \mu \mathrm{m}$ bands for sake of relative simplicity of the iterative computations to fit the data with the model. In Perrin et al. (2003) the model has been simplified to allow quicker computations hence allowing more parameters in the fit. Thanks to this choice, simultaneous fit of the visibilities and of the photometric data has become possible, thus more reliably fixing the temperatures of the star and of the layer which would otherwise be coupled in the visibility only fit. Optical depths are then varied between each bands to refine the adjustment of visibilities. Several species $(\mathrm{CO}, \mathrm{H} 2 \mathrm{O}, \mathrm{SiO}, \mathrm{OH}-, \ldots)$ may contribute to the opacity in these bands in different ways and one may expect to find different optical depths in the different bands.

This model can account for both the Gaussian-like visibility curves and the variation of apparent size with wavelength. A simple interpretation of the IR visibility curves lead to an overestimation of the photospheric size.

The observations of the $\mathrm{SiO}$ masers presented here should help test this model.

The following sections will describe the $\mathrm{SiO}$ maser observations and the data analysis applied. Then, the results are given in terms of the velocity averaged images and a discussion of individual stars. Particular attention is paid to R Aquarii which appears to be rotating and exhibits an equatorial molecular disk. Following is a comparison of the $\mathrm{SiO}$ maser and infrared measurements and then a discussion of the variation of the observed rings with stellar pulsation phase. Finally, we consider rotation of the envelope and the magnetic field structure.

\section{Observations and data reduction}

The observations were obtained in four 18 hour sessions on 25 Jan., 29 Apr., 4 Aug. and 10 Nov. 2001. Two 4 MHz wide channels in each right- and left-hand circular polarization were recorded at 42.820587 and $43.122027 \mathrm{GHz}$ to cover the $v=2$, $J=1-0$ and $v=1, J=1-0$ transitions of SiO. Two bit sampling was used in the recording. The correlations resulted in 128 channels in each of the combinations of right- and leftcircular polarization for each transition. A strong, nearby continuum source was observed before each star to serve as delay, bandpass and polarization calibrator. The observations are summarized in Table 1 which lists the stars, calibrators, central velocities and the dates observed; unless otherwise noted, the IR data are from Mennesson et al. (2002) and are uniform disk model diameter fits. The calibrators are all quasars with milliarcsecond accuracy positions; the Hipparcos (ESA 1997) positions of the stars, evaluated at the epoch of the observations, were used. Note: the center velocities were picked to center the spectra in the observing band and are not necessarily the systemic velocities. Continuum calibration followed the usual procedure for delay, polarization calibration (Cotton 1993). Data analysis was done in the NRAO AIPS package. Measured system temperatures and assumed antenna gains were used to calibrate the amplitudes of the continuum sources. Each continuum calibrator was observed with the same velocity offset as its corresponding star. All calibrators were amplitude and phase self-calibrated.

Linear polarization calibration was based on monitoring of the continuum calibrators from the VLA. Weakly polarized 
Table 1. Miras observed.

\begin{tabular}{|c|c|c|c|c|c|c|c|c|}
\hline Star & Calibrator & $\begin{array}{r}\mathrm{UD}_{2.2 \mu \mathrm{m}} \\
(\mathrm{mas}) \\
\end{array}$ & $\begin{array}{r}\mathrm{UD}_{3.6 \mu \mathrm{m}} \\
\text { (mas) } \\
\end{array}$ & $\begin{array}{r}\text { Dust }_{11 \mu \mathrm{m}}^{1} \\
\text { (mas) }\end{array}$ & $\begin{array}{r}\text { Vel A }{ }^{2} \\
\left(\mathrm{~km} \mathrm{~s}^{-1}\right) \\
\end{array}$ & $\begin{array}{r}\text { Vel B } \\
\left(\mathrm{km} \mathrm{s}^{-1}\right) \\
\end{array}$ & Period & Sessions $^{4}$ \\
\hline R Andromadae & $\mathrm{J} 0136+4751$ & & & & -14.8 & -14.8 & 409.33 & $\mathrm{AB}$ \\
\hline omicron Ceti & $\mathrm{J} 0217+0144$ & $24.40(0.11)$ & $35.2(2.0)$ & 120 & 48.0 & 45.0 & 331.96 & $\mathrm{ABCD}$ \\
\hline U Orionis & J0555+3948 & $15.59(0.06)$ & $28.8(0.1)$ & 160 & -34.0 & -40.0 & 368.30 & $\mathrm{ABCD}$ \\
\hline R Leonis & J0854+2006 & $29.43^{5}(0.05)$ & $36.0(0.5)$ & 140 & 2.0 & -1.0 & 309.95 & $\mathrm{ABCD}$ \\
\hline W Hydrae & $\mathrm{J} 1337-1257$ & & & & 41.7 & 41.7 & 361.00 & $\mathrm{C}$ \\
\hline S Coronae Boralis & $\mathrm{J} 1613+3412$ & $11.35^{6}(0.26)$ & & & 4.0 & 3.0 & 360.26 & $\mathrm{ABCD}$ \\
\hline U Herculis & $\mathrm{J} 1613+3412$ & $10.98(0.01)$ & $14.3(0.5)$ & & -14.0 & -16.0 & 406.10 & $\mathrm{ABCD}$ \\
\hline R Aquarii & $\mathrm{J} 2334+0736$ & $16.88(0.56)$ & $34.3(1.0)$ & 140 & -23.0 & -23.0 & 386.96 & $\mathrm{AB}$ \\
\hline R Cassopeiae & $\mathrm{J} 2322+5057$ & $24.78(0.09)$ & $31.1(3.0)$ & & 25.4 & 25.4 & 430.46 & $\mathrm{CD}$ \\
\hline
\end{tabular}

${ }^{1}$ Fitted inner dust shell diameter from Danchi et al. (1994).

${ }^{2} v=2, J=1-0$ transition of $\mathrm{SiO}$ at $42.8 \mathrm{GHz}$.

${ }^{3} v=1, J=1-0$ transition of $\mathrm{SiO}$ at $43.1 \mathrm{GHz}$.

${ }^{4}$ A $=25$ Jan. 2001, B = 29 Apr. 2001, C = 4 Aug. 2001, D = 10 Nov. 2001.

${ }^{5}$ From Perrin et al. (1999).

${ }^{6}$ From van Belle et al. (2002).

calibrators were used jointly to determine the instrumental calibration and the more polarized sources were used to calibrate the polarization angle. The observed polarization angle was determined by summed Stokes $Q$ and $U$ flux densities from imaging.

Bandpass calibration used only continuum calibrator observations made with the same velocity settings as the line data. Delay calibration used fringe fits of the continuum calibrator sources with the fitted rates set to zero. This procedure, together with accurate positions of the calibrators and targets, resulted in accurate relative registration of the images derived in the two transitions. Since only approximate Doppler tracking was used during the observations, the cross correlation spectra were interpolated to a common velocity.

Amplitude calibration of the stellar observations was based on the standard template fitting technique for sources with good signal-to-noise ratio and system temperatures and antenna gains for the weaker sources. Phase calibration was done by self calibrating a spectral channel (or an average of several channels) which contained relatively strong emission in simple structure. In order to obtain the maximum dynamic range possible, this procedure was occasionally used to bootstrap the calibration of a channel with strong but complex emission which was then used for the phase calibration. This phase calibration was applied to all channels in both transitions. This procedure results in proper alignment of all images of a given star at a particular epoch.

The stars were observed with multiple snapshots which results in relatively limited uv-coverage given the large size and sometimes complex structure in a given channel. In order to minimize the effects of the limited uv-coverage, CLEANing was limited to regions selected manually which appeared to have true emission, based on a preliminary channel-by-channel CLEAN. After the set of CLEAN boxes were selected, all channels were imaged and CLEANed in Stokes $I, Q$, and $U$.

The result of the poor uv-coverage is limited dynamic range in the images. Channel images with bright components include artifacts which are significantly stronger than real features in channels with less emission. A simple summation over velocity including these artifacts will mask most of the weak, but real, features in the image. Once three-dimensional images were obtained, they were compressed to two-dimensional in Stokes I by taking the maximum value along the velocity axis subject to a lower limit set by a multiple of the off source rms noise and a fraction of the peak in the velocity image. This suppresses random noise values and artifacts resulting from the low dynamic range. The lower limits depended on the image but were typically of order five percent of the peak and three times the rms noise. A similar procedure was used for Stokes $Q$ and $U$ except that there was no lower limit on the value and the sum of pixel values over velocity were used.

The maser spots occur in an extended, possible noncircular, ring around the star. However, the number and distribution of spots is variable and generally insufficient to determine a model more complex that a circular diameter and ring thickness. Even with this approximation, joint analysis of the two transitions was needed to determine the center of the ring.

The diameters and widths of the $\mathrm{SiO}$ maser rings were determined from a moment analysis of compressed (2D) Stokes $I$ images. In this analysis, values below several times the noise level were excluded and the pixel values were summed in velocity. The maser ring was assumed to be circular and the center was determined by a direct parameter search from a joint analysis of the images in the two transitions. For each trial position, the image was collapsed into a single dimension being the average flux density as a function of distance from the trial center. The first moment of this function was taken as the trial ring radius and the second moment about this radius was determined independently for the two transitions. The location determined to the center of the ring was the one that minimized the sum of the second moments, i.e. gives the narrowest width of the rings. An example is shown in Fig. 1.

The velocity fields evident in the derived images are complex and change as individual maser spots brighten and fade. In order to look for evidence of systematic rotation in the 


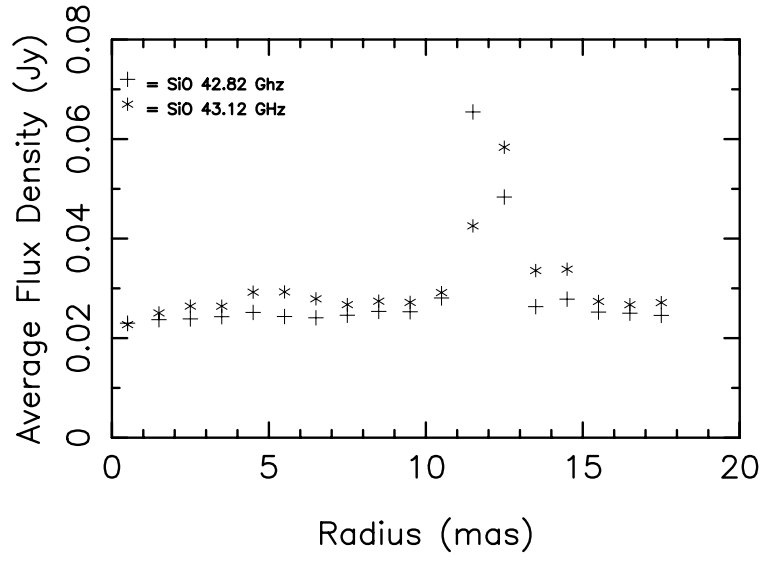

Fig. 1. Stokes' I flux density averages per unit area in annuli around the center of U Orionis demonstrating the concentration of the maser emission into a ring.

masing region, flux density weighted averages of the velocity were made in sectors around the maser ring with a width twice the second moment widths.

\section{Results}

The compressed total intensity images for each epoch and transition with superposed polarization "E" vectors are shown in Figs. 2-10. The fitted ring diameters and width are shown in Table 2 and in Figs. 11-13 as well as in Figs. 2-10. Each row in Table 2 corresponds to a given star and transition and the measured ring diameter and width for each session are given in separate columns.

The sector averaged velocities are given in Table 3 and the corresponding summed flux densities are given in Table 4. In both these tables, a row corresponds to observations of a single star at a single epoch and the different sectors and transitions are given in separate columns. The column headings give the position angle in degrees of the center of the sector.

\section{Discussion}

\section{1. $R$ Andromadae}

Only a couple of maser spots were ever detected from this star.

\subsection{Omicron Ceti (Mira)}

Danchi et al. (1994) measured the radius of the inner radius of

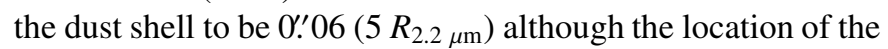
inner edge of the dust shell may be time variable. Omicron Ceti was observed in all four sessions reported in this paper; see Figs. 3 and 11. The diameter of the ring in this star is approximately half that of the inner dust diameter reported by Danchi et al. (1994).

\subsection{U Orionis}

Danchi et al. (1994) measured the radius of the inner radius of the dust shell to be $0.08\left(10 R_{2.2} \mu \mathrm{m}\right)$ and an outer radius

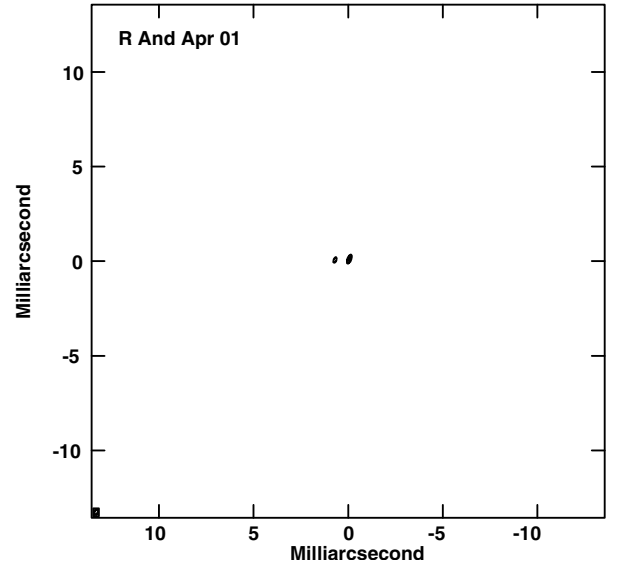

Fig. 2. R Andromadae. $v=1, J=1-0$ transition of $\mathrm{SiO}$ at $43.1 \mathrm{GHz}$.

of 2'.5. This star was observed in all four sessions reported in this paper and shows remarkably little variations in ring diameter; see Figs. 4 and 11. The size at $3.6 \mu \mathrm{m}$ wavelength reported by Mennesson et al. (2002) (see Fig. 11) is approximately the same as the diameter of the $\mathrm{SiO}$ ring.

\subsection{R Leonis}

Previous VLBI observations of $\mathrm{R}$ Leonis were made by Colomer et al. (1992); a lunar occultation experiment is reported by Cernicharo et al. (1994). Danchi et al. (1994) measured the radius of the inner radius of the dust shell to be $0 .{ }^{\prime} 07$ $\left(5 R_{2.2} \mu \mathrm{m}\right)$. R Leonis was observed in all four sessions reported in this paper; see Figs. 5 and 12.

\subsection{W Hydrae}

Observations of the $\mathrm{SiO}$ masers in $\mathrm{W}$ Hydrae have been reported by Lane et al. (1980). This star was only observed in the August 2001 session.

\subsection{S Coronae Boralis}

S Coronae Boralis was observed in all four sessions reported in this paper; see Figs. 7 and 12.

\subsection{U Herculis}

Observations by Colomer et al. (1992) failed to detect the $\mathrm{SiO}$ emission in U Herculis. Diamond et al. (1994) demonstrated that the masers in U Herculis lay in a circular ring of average diameter $\sim 19$ milliarcsec. Diamond \& Kemball (1998) reported on 4 epochs of polarization imaging of $U$ Her taken over the period from June 1995 - April 1996. During this time the average radius of the $\mathrm{SiO}$ ring varied over the range $\sim 11.5 \rightarrow 12.7$ milliarcsec. The polarization structure was observed to have a complex nature. This star was observed in all four sessions reported in this paper; see Figs. 8 and 13. 
Table 2. SiO ring diameters.

\begin{tabular}{lrrrrrrr}
\hline \hline & $\begin{array}{r}\text { Jan. 01 (A) } \\
\text { Diam. (mas) }\end{array}$ & Width & $\begin{array}{r}\text { Apr. 01 (B) } \\
\text { Diam. (mas) }\end{array}$ & Width & $\begin{array}{r}\text { Aug. 01 (C) } \\
\text { Diam. (mas) }\end{array}$ & Width & $\begin{array}{c}\text { Nov. 01 (D) } \\
\text { Diam. (mas) }\end{array}$ \\
Source ${ }^{1}$ & 73.0 & 6.0 & 72.4 & 4.7 & 60.0 & 4.3 & 75.6 \\
Width
\end{tabular}

${ }^{1}$ [1] denotes $v=1, J=1-0$ transition of $\mathrm{SiO}$ at $43.1 \mathrm{GHz}$. [2] denotes $v=2, J=1-0$ transition of $\mathrm{SiO}$ at $42.8 \mathrm{GHz}$.
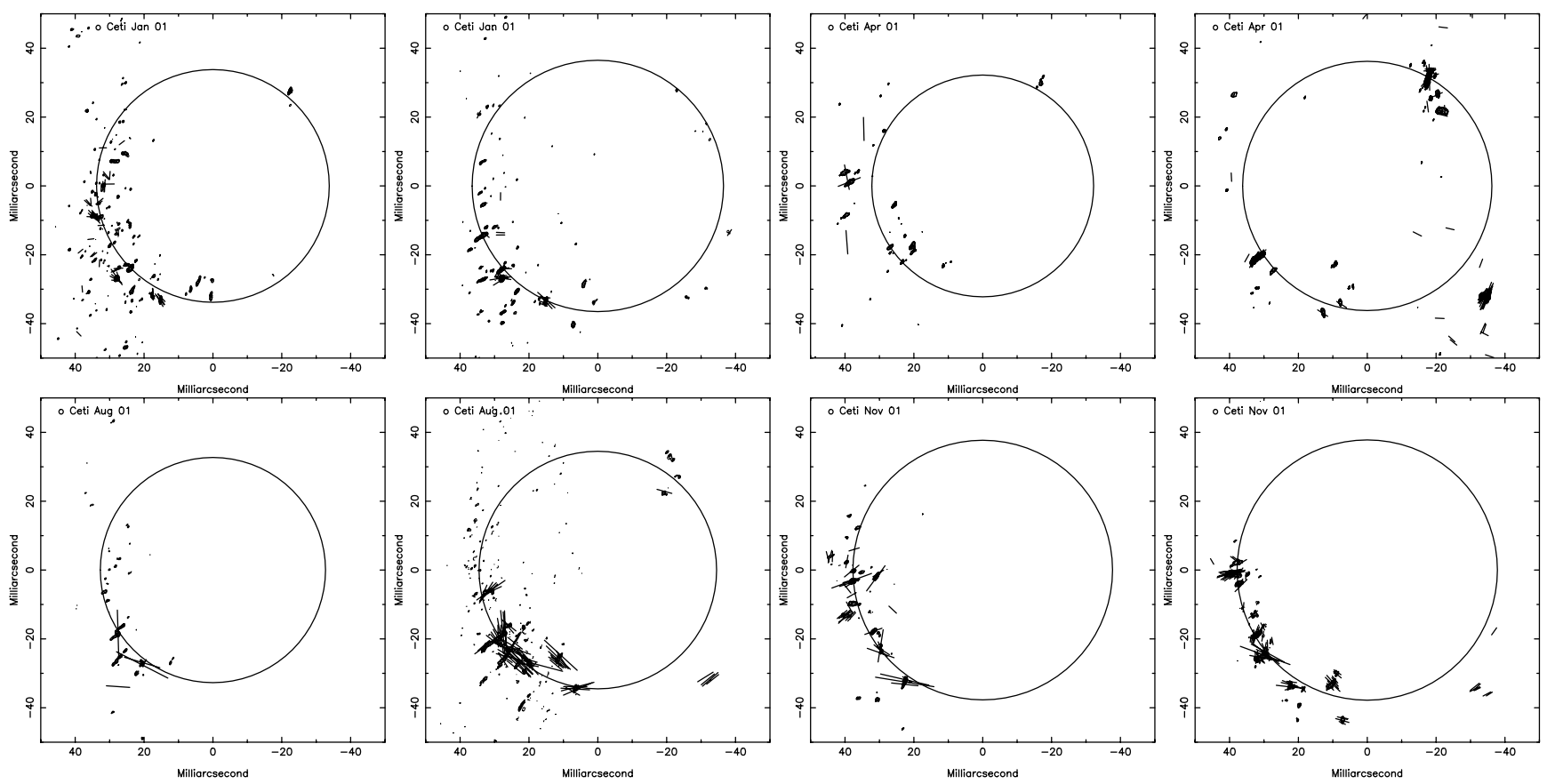

Fig. 3. Omicron Ceti (Mira). Odd columns are $v=2, J=1-0$ transition of $\mathrm{SiO}$ at $42.8 \mathrm{GHz}$, even columns are $v=1, J=1-0$ transition of $\mathrm{SiO}$ at 43.1 GHz. Polarization "E" vectors are over-plotted on contours of total intensity. Circles show the fitted rings as given in Table 2.

\subsection{R Aquarii}

Danchi et al. (1994) measured the inner radius of the dust shell to be $0.07\left(8 R_{2.2} \mu \mathrm{m}\right)$ at luminosity phase 0.7 . Apparent contraction of the $\mathrm{SiO}$ maser shell in $\mathrm{R}$ Aquarii was reported by Boboltz et al. (1997). This star is in a symbiotic system and exhibits a jet seen both optically and in the radio (Hollis et al. 1999) apparently emanating from an accretion disk around the hot companion star. Based on VLBA observations a few months prior to the first epoch reported here, Hollis et al. (2001) claim evidence of differential rotation in the maser shell with an axis of rotation at a position angle of approximately $150^{\circ}$.

R Aquarii was observed only in the January and April 2001 sessions; see Figs. 9 and 13. The size at $3.6 \mu \mathrm{m}$ wavelength reported by Mennesson et al. (2002) (see Fig. 13) is approximately the same as the diameter of the $\mathrm{SiO}$ ring. The $\mathrm{SiO}$ maser data in Fig. 9 show curious extended linear features on opposite sides of the ring structure. The velocities in these features are the furthest from the average (systemic?) on the end closest to the star, consistent with the differential rotation reported by 
Table 3. Sector averaged velocities $\left(\mathrm{km} \mathrm{s}^{-1}\right)$.

\begin{tabular}{|c|c|c|c|c|c|c|c|c|c|c|c|c|c|c|c|c|}
\hline \multirow{2}{*}{$\begin{array}{r}\text { Source } \\
\text { epoch }\end{array}$} & \multirow[b]{2}{*}{$23^{\circ}$} & \multirow[b]{2}{*}{$68^{\circ}$} & \multicolumn{6}{|c|}{$[1]^{1}$} & \multicolumn{8}{|c|}{$[2]^{1}$} \\
\hline & & & $113^{\circ}$ & $158^{\circ}$ & $203^{\circ}$ & $248^{\circ}$ & $293^{\circ}$ & $338^{\circ}$ & $23^{\circ}$ & $68^{\circ}$ & $113^{\circ}$ & $158^{\circ}$ & $203^{\circ}$ & $248^{\circ}$ & $293^{\circ}$ & $338^{\circ}$ \\
\hline o Ceti A & 1.5 & 0.2 & -1.6 & -0.6 & -2.9 & -4.0 & 4.8 & 2.0 & 1.9 & -0.1 & 0.2 & 1.8 & -0.1 & 4.4 & 2.3 & 3.0 \\
\hline B & -2.7 & -2.0 & -2.3 & -1.4 & 4.3 & 4.4 & 6.1 & -0.1 & -1.5 & -2.2 & -3.8 & 0.6 & 0.1 & 0.1 & 1.2 & -0.1 \\
\hline $\mathrm{C}$ & -5.2 & -2.3 & -3.2 & -2.9 & 4.7 & -5.2 & 6.1 & 4.4 & -1.2 & -2.7 & -3.2 & -1.4 & 2.1 & 1.1 & 1.3 & 1.4 \\
\hline D & -0.4 & -2.3 & -2.6 & 0.3 & 1.6 & -4.6 & 1.8 & 0.7 & -0.1 & -1.6 & -2.1 & -2.0 & 4.1 & 4.6 & 4.9 & 5.3 \\
\hline U Ori A & 1.0 & -1.2 & -0.6 & & -1.6 & -2.7 & -4.4 & -2.5 & 2.6 & 1.8 & 0.6 & -1.3 & -1.0 & -1.7 & -0.5 & \\
\hline B & & 1.0 & 0.1 & 3.3 & -1.3 & -1.5 & -0.9 & -3.6 & & 1.6 & 0.3 & -0.9 & & -2.3 & -3.3 & 1.5 \\
\hline $\mathrm{C}$ & 1.0 & -3.0 & -0.3 & 0.0 & 0.7 & -3.5 & -2.6 & -1.7 & -0.5 & -0.9 & -0.2 & -2.0 & -2.2 & -1.1 & -0.2 & -1.2 \\
\hline D & -5.2 & -1.5 & -0.3 & -2.0 & -1.2 & -3.7 & -1.0 & -1.6 & -1.3 & -1.2 & 0.2 & -2.8 & -2.5 & -0.6 & -0.6 & -0.3 \\
\hline R Leo A & 1.7 & -3.5 & -0.9 & -3.1 & 0.8 & -5.1 & -0.6 & 0.0 & 4.3 & 2.5 & 0.8 & -1.1 & -3.6 & -3.1 & 2.3 & 2.4 \\
\hline B & 0.0 & 1.3 & 1.2 & 5.3 & -2.4 & -0.6 & 3.4 & 3.0 & 0.3 & 2.2 & 1.7 & 2.3 & 6.7 & 0.0 & 4.3 & 3.4 \\
\hline $\mathrm{C}$ & -1.5 & 2.9 & 1.9 & 5.0 & -5.7 & -5.2 & -2.8 & -5.2 & 3.5 & 3.6 & 4.1 & 5.6 & 8.3 & -2.4 & -2.4 & 8.8 \\
\hline D & 1.4 & 2.7 & 4.1 & -0.1 & -3.6 & -2.9 & -2.0 & -4.6 & -1.1 & 3.1 & 0.7 & -2.7 & -1.7 & -1.8 & -0.6 & -2.9 \\
\hline W Hya C & 0.4 & -3.1 & -2.5 & -1.5 & -3.4 & -1.6 & -1.0 & -1.8 & 1.6 & 3.2 & -4.9 & -1.1 & 0.6 & 0.2 & -1.0 & -2.1 \\
\hline $\mathrm{S}$ CrB A & 2.1 & 3.2 & 2.6 & 0.6 & -4.4 & -1.8 & -2.8 & -0.2 & 3.7 & 3.0 & 5.7 & -2.5 & -2.8 & 0.6 & -0.7 & 1.8 \\
\hline B & -4.8 & 4.6 & 3.8 & 0.7 & -4.5 & -0.4 & -2.8 & -0.6 & -4.0 & -2.2 & & -1.6 & -4.1 & 1.1 & -1.7 & 0.4 \\
\hline $\mathrm{C}$ & -4.3 & 3.0 & 2.0 & -4.1 & -4.7 & -4.5 & -2.7 & -0.3 & 0.0 & -4.8 & -0.7 & -4.9 & -2.2 & -2.3 & -6.4 & \\
\hline $\mathrm{D}$ & -3.4 & 1.6 & -0.4 & -4.5 & -5.8 & -2.4 & -2.9 & -2.0 & -1.1 & & 0.5 & -3.1 & -4.1 & -3.0 & & -0.6 \\
\hline U Her A & 2.3 & 1.6 & 1.7 & -1.0 & 0.4 & 6.0 & -1.7 & 3.4 & 4.7 & 3.8 & 3.7 & 1.6 & 4.6 & 3.0 & -2.2 & 6.6 \\
\hline B & 0.8 & 1.3 & 1.2 & -1.2 & 0.3 & -1.2 & -3.8 & 3.6 & 3.8 & 3.0 & 2.0 & -1.9 & -1.9 & 0.0 & -0.4 & 4.7 \\
\hline $\mathrm{C}$ & 0.2 & 3.2 & 1.6 & -3.6 & 1.4 & -3.8 & 0.5 & 3.4 & 0.9 & 1.4 & 2.4 & 3.5 & 2.5 & 1.7 & -0.7 & 3.0 \\
\hline D & 0.8 & 3.1 & 1.5 & -2.7 & -2.0 & -0.6 & -1.1 & 3.2 & 3.2 & 3.6 & 2.7 & 2.8 & 1.2 & -0.3 & -0.6 & 0.8 \\
\hline R Aqr A & 0.8 & 4.4 & 4.9 & -5.5 & -5.5 & -3.7 & -3.1 & -1.9 & -0.3 & & & -5.7 & -4.9 & -3.2 & -1.8 & -0.9 \\
\hline B & 0.4 & 5.2 & 4.1 & -5.6 & -4.3 & -3.1 & -3.4 & -1.4 & -1.6 & 5.6 & 4.7 & -4.9 & -4.3 & -3.4 & -1.0 & -1.2 \\
\hline R Cas C & 1.1 & -1.9 & 1.4 & -0.9 & -1.4 & -1.0 & -0.4 & 0.8 & 1.0 & 1.5 & 1.3 & 2.2 & -0.6 & -0.2 & -0.2 & 2.6 \\
\hline D & -0.4 & -3.7 & -0.8 & -1.8 & 1.3 & -0.3 & -0.1 & 3.6 & 1.4 & 0.7 & 0.9 & -2.4 & -0.6 & 0.2 & -0.7 & 2.9 \\
\hline
\end{tabular}

${ }^{1}$ [1] denotes $v=1, J=1-0$ transition of $\mathrm{SiO}$ at $43.1 \mathrm{GHz}$. [2] denotes $v=2, J=1-0$ transition of $\mathrm{SiO}$ at $42.8 \mathrm{GHz}$.
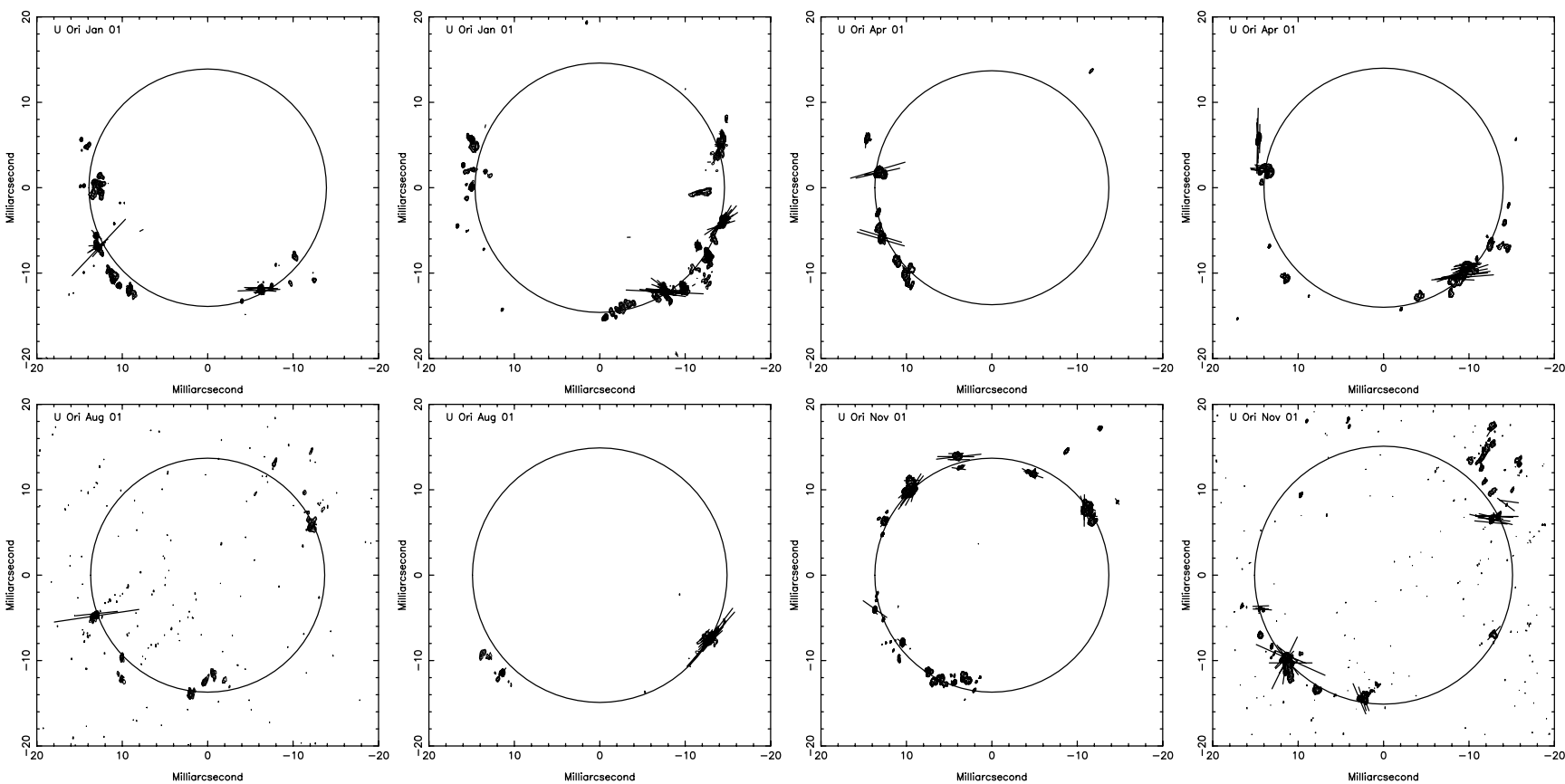

Fig. 4. U Orionis Odd columns are $v=2, J=1-0$ transition of $\mathrm{SiO}$ at $42.8 \mathrm{GHz}$, even columns are $v=1, J=1-0$ transition of $\mathrm{SiO}$ at 43.1 GHz. Polarization "E" vectors are over-plotted on contours of total intensity. Circles show the fitted rings as given in Table 2.

Hollis et al. (2001). Figure 14 shows the total intensity contours with inset plots of the radial velocity giving the trend towards the mean away from the star. Furthermore, these two features appear to be aligned with the center of the star. This suggests a differentially rotating disk in the equatorial plane of the star. If so, then this feature is transient as it was not visible in the 
Table 4. Sector summed flux densities (Jy).

\begin{tabular}{|c|c|c|c|c|c|c|c|c|c|c|c|c|c|c|c|c|}
\hline $\begin{array}{r}\text { Star } \\
\text { epoch }\end{array}$ & $23^{\circ}$ & $68^{\circ}$ & $113^{\circ}$ & $158^{\circ}$ & $\begin{array}{c}{[1]^{1}} \\
203^{\circ}\end{array}$ & $248^{\circ}$ & $293^{\circ}$ & $338^{\circ}$ & $23^{\circ}$ & $68^{\circ}$ & $113^{\circ}$ & $\begin{array}{c}{[2]^{1}} \\
158^{\circ}\end{array}$ & $203^{\circ}$ & $248^{\circ}$ & $293^{\circ}$ & $338^{\circ}$ \\
\hline o Ceti A & 3 & 12 & 51 & 26 & 6 & 5 & 7 & 7 & 4 & 18 & 33 & 39 & 5 & 2 & 4 & 54 \\
\hline B & 2 & 2 & 18 & 6 & 1 & 1 & 6 & 34 & 2 & 5 & 6 & 2 & 2 & 2 & 2 & 5 \\
\hline $\mathrm{C}$ & 2 & 30 & 63 & 54 & 4 & 4 & 8 & 15 & 7 & 14 & 34 & 27 & 2 & 2 & 2 & 2 \\
\hline D & 11 & 88 & 165 & 69 & 4 & 15 & 12 & 12 & 5 & 50 & 113 & 129 & 3 & 3 & 3 & 3 \\
\hline U Ori A & 1 & 15 & 9 & & 32 & 26 & 22 & 1 & 1 & 20 & 56 & 11 & 49 & 5 & 1 & \\
\hline B & & 58 & 6 & 2 & 51 & 24 & 2 & 2 & & 318 & 52 & 17 & & 2 & 2 & 2 \\
\hline $\mathrm{C}$ & 1 & 1 & 1 & 1 & 2 & 14 & 1 & 1 & 1 & 1 & 23 & 8 & 2 & 1 & 7 & 1 \\
\hline D & 1 & 2 & 136 & 42 & 2 & 8 & 31 & 2 & 207 & 66 & 44 & 23 & 3 & 7 & 107 & 23 \\
\hline R Leo A & 150 & 69 & 5 & 123 & 3 & 16 & 4 & 15 & 341 & 392 & 3 & 87 & 3 & 31 & 144 & 211 \\
\hline B & 395 & 56 & 806 & 6 & 5 & 6 & 242 & 62 & 1881 & 241 & 1651 & 209 & 6 & 6 & 604 & 125 \\
\hline $\mathrm{C}$ & 15 & 10 & 41 & 5 & 1 & 1 & 3 & 4 & 3 & 11 & 33 & 10 & 1 & 5 & 21 & 1 \\
\hline D & 1 & 16 & 59 & 2 & 5 & 1 & 237 & 3 & 1 & 29 & 42 & 59 & 80 & 15 & 2 & 1 \\
\hline W Hya C & 180 & 124 & 79 & 549 & 184 & 96 & 665 & 792 & 582 & 109 & 16 & 232 & 988 & 16 & 620 & 351 \\
\hline $\mathrm{S}$ CrB A & 5 & 6 & 36 & 3 & 17 & 30 & 83 & 10 & 4 & 9 & 11 & 3 & 8 & 21 & 96 & 9 \\
\hline B & 1 & 6 & 8 & 1 & 4 & 5 & 9 & 3 & 1 & 1 & & 1 & 3 & 6 & 9 & 2 \\
\hline $\mathrm{C}$ & 1 & 1 & 1 & 6 & 15 & 2 & 2 & 1 & 5 & 1 & 1 & 41 & 3 & 5 & 1 & \\
\hline D & 2 & 34 & 2 & 71 & 13 & 108 & 40 & 3 & 39 & & 3 & 191 & 8 & 23 & & 3 \\
\hline U Her A & 21 & 12 & 25 & 37 & 4 & 17 & 114 & 91 & 150 & 6 & 20 & 55 & 3 & 35 & 59 & 22 \\
\hline B & 3 & 7 & 17 & 10 & 4 & 29 & 45 & 81 & 2 & 134 & 73 & 17 & 3 & 85 & 40 & 78 \\
\hline $\mathrm{C}$ & 2 & 13 & 50 & 6 & 2 & 2 & 40 & 107 & 2 & 2 & 39 & 4 & 3 & 2 & 42 & 21 \\
\hline D & 3 & 24 & 116 & 13 & 3 & 39 & 83 & 306 & 2 & 20 & 65 & 4 & 6 & 65 & 219 & 75 \\
\hline R Aqr A & 9 & 185 & 53 & 26 & 33 & 462 & 57 & 28 & 11 & & & 24 & 35 & 601 & 18 & 36 \\
\hline B & 2 & 43 & 6 & 15 & 50 & 120 & 18 & 25 & 8 & 12 & 3 & 7 & 32 & 113 & 2 & 89 \\
\hline R Cas C & 2 & 4 & 105 & 4 & 4 & 28 & 55 & 9 & 4 & 35 & 4 & 5 & 11 & 41 & 14 & 13 \\
\hline D & 7 & 22 & 55 & 19 & 2 & 96 & 35 & 21 & 43 & 25 & 11 & 18 & 20 & 86 & 29 & 25 \\
\hline
\end{tabular}

${ }^{1}$ [1] denotes $v=1, J=1-0$ transition of $\mathrm{SiO}$ at $43.1 \mathrm{GHz}$. [2] denotes $v=2, J=1-0$ transition of $\mathrm{SiO}$ at $42.8 \mathrm{GHz}$.
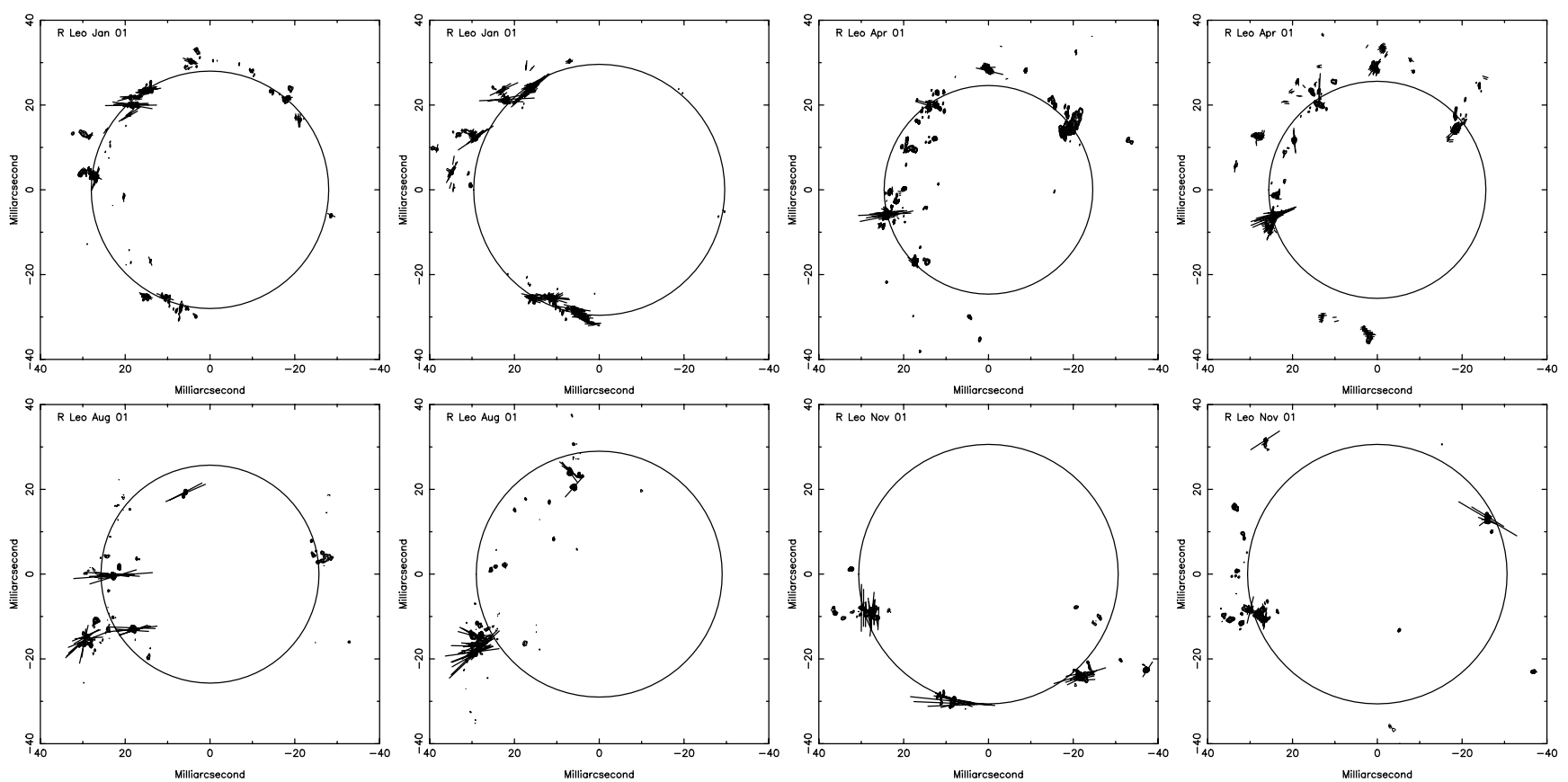

Fig. 5. R Leonis. Odd columns are $v=2, J=1-0$ transition of $\mathrm{SiO}$ at $42.8 \mathrm{GHz}$, even columns are $v=1, J=1-0$ transition of SiO at 43.1 GHz. Polarization "E" vectors are over-plotted on contours of total intensity. Circles show the fitted rings as given in Table 2.

images presented by Hollis et al. (2001) and is much less prominent in our April 2001 observations.

If the measurements shown in Fig. 14 are in fact dominated by rotational velocity, the rotational axis of the star is in the plane of the sky and its distance is $200 \mathrm{pc}$, then the rotation period at the center of the $\mathrm{SiO}$ ring is 22 years. If the dominant maser features in Fig. 14 are in the equatorial plane, then the 

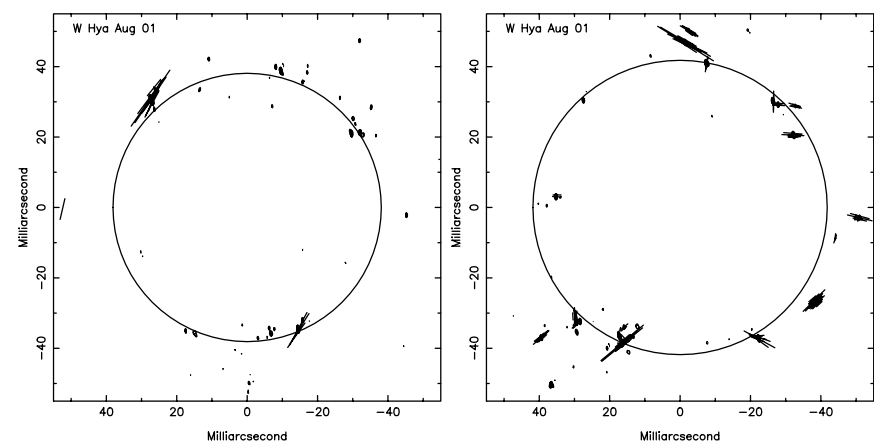

Fig. 6. W Hydrae. Left is $v=2, J=1-0$ transition of $\mathrm{SiO}$ at $42.8 \mathrm{GHz}$, right is $v=1, J=1-0$ transition of $\mathrm{SiO}$ at $43.1 \mathrm{GHz}$. Polarization "E" vectors are over-plotted on contours of total intensity. Circles show the fitted rings as given in Table 2.
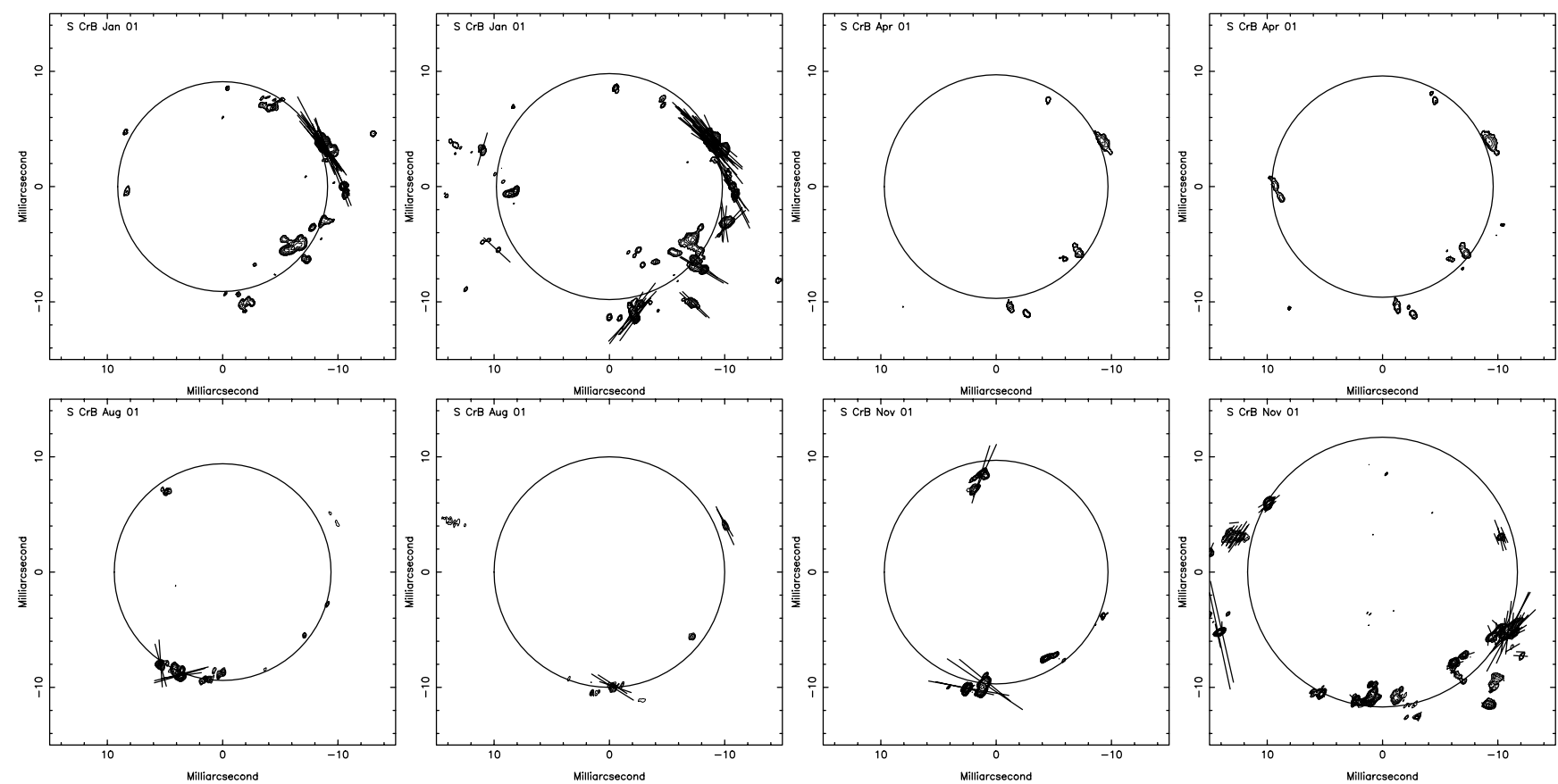

Fig. 7. S Coronae Boralis. Odd columns are $v=2, J=1-0$ transition of $\mathrm{SiO}$ at $42.8 \mathrm{GHz}$, even columns are $v=1, J=1-0$ transition of $\mathrm{SiO}$ at $43.1 \mathrm{GHz}$. Polarization "E" vectors are over-plotted on contours of total intensity. Circles show the fitted rings as given in Table 2.

projected direction of the pole is at position angle $-10^{\circ} / 170^{\circ}$; the direction inferred by Hollis et al. (2001) was $-30^{\circ} / 150^{\circ}$.

A rotation model was fitted to the velocity field measured along the slice in Fig. 14 by a direct parameter search. The velocity at the ring diameter of 16.3 mas was $3.92 \mathrm{~km} \mathrm{~s}^{-1}$ and scaled with distance from the star as $r^{-1.81}$. The data and model are shown in Fig. 15. This radial scaling of velocity is much faster than the -0.5 expected from a pure Keplerian velocity field. The remarkable symmetry in emission and velocity field in Figs. 14 and 15 strongly argue that these masers are from a differentially rotating equatorial disk of material. The variation in velocity with radius may be due to a transition from a corotating inner atmosphere to a non rotating outer envelope. The disk-like feature is less well defined in the April 2001 observations but the velocity structure is very similar.

There is no evidence of the contraction of the $\mathrm{SiO}$ maser ring as previously reported by Boboltz et al. (1997). However, this is not unexpected as the monitoring program of TX Cam (Diamond \& Kemball, in preparation) demonstrates that the mode of structural change can vary on time-scales of a few months to a year.

\section{9. $R$ Cassopeiae}

The first reported VLBI observations of the $\mathrm{SiO}$ masers around R Cassopeiae were reported by Moran et al. (1979). Subsequent VLBI observations have been reported by McIntosh et al. (1989), Colomer et al. (1992), Colomer et al. (1996), Phillips et al. (2001) and Phillips et al. (2003). This star was only observed in the August and November 2001 sessions; see Fig. 10. The source is very heavily resolved and the ring is defined by a few spots.

\subsection{Comparison of SiO and IR diameters}

The 2.2 and $3.6 \mu \mathrm{m}$ stellar diameters are not well sampled in luminosity phase so only average values can be compared. Both the 2.2 and $3.6 \mu \mathrm{m}$ apparent sizes are believed to be affected 

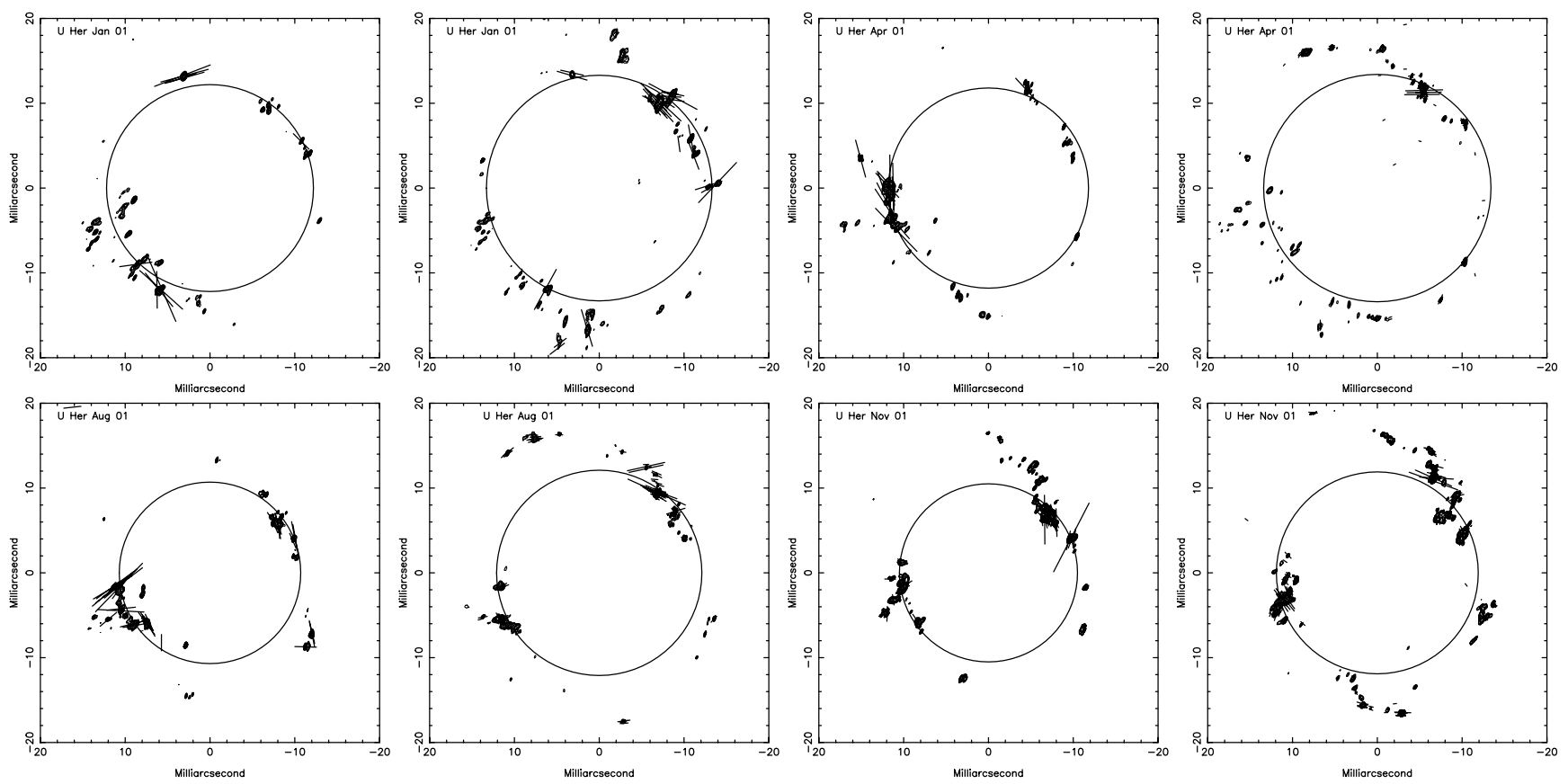

Fig. 8. U Herculis. Odd columns are $v=2, J=1-0$ transition of $\mathrm{SiO}$ at $42.8 \mathrm{GHz}$, even columns are $v=1, J=1-0$ transition of $\mathrm{SiO}$ at $43.1 \mathrm{GHz}$. Polarization "E" vectors are over-plotted on contours of total intensity. Circles show the fitted rings as given in Table 2.
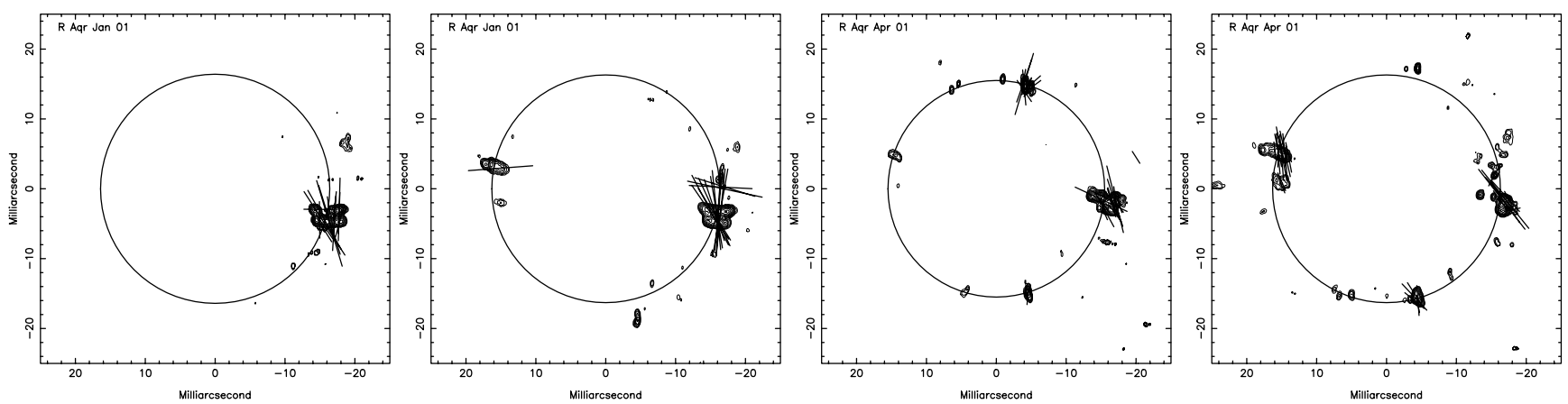

Fig. 9. R Aquarii. Odd columns are $v=2, J=1-0$ transition of $\mathrm{SiO}$ at $42.8 \mathrm{GHz}$, even columns are $v=1, J=1-0$ transition of $\mathrm{SiO}$ at $43.1 \mathrm{GHz}$. Polarization "E" vectors are over-plotted on contours of total intensity. Circles show the fitted rings as given in Table 2.
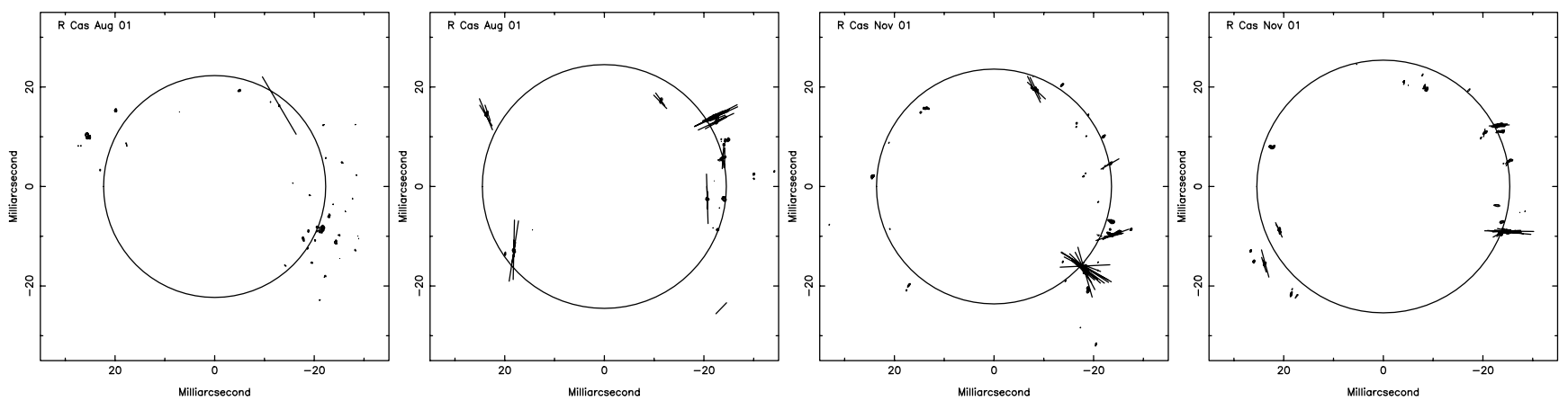

Fig. 10. R Cassopeiae. Odd columns are $v=2, J=1-0$ transition of $\mathrm{SiO}$ at $42.8 \mathrm{GHz}$, even columns are $v=1, J=1-0$ transition of $\mathrm{SiO}$ at 43.1 GHz. Polarization "E" vectors are over-plotted on contours of total intensity. Circles show the fitted rings as given in Table 2.

by opacity and temperature effects in the stellar envelope, the $3.6 \mu \mathrm{m}$ apparent sizes more so than the $2.2 \mu \mathrm{m}$ sizes. Recent modeling by Perrin et al. (2003) suggest that the apparent size at $2.2 \mu \mathrm{m}$ is a third larger than the photospheric diameter.

Table 5 lists the ratios of the $\mathrm{SiO}$ maser ring sizes to the $2.2 \mu \mathrm{m}$ diameters. In this table the first column is the name of the star, the second is the mean diameter of the ring in the $v=1, J=1-0$ transition followed by the mean in units of the $2.2 \mu \mathrm{m}$ diameters from Table 1 , the rms about this mean and the percentage fluctuations. The final four columns are the mean in mas, the mean in $2.2 \mu \mathrm{m}$ diameters, the rms and percentage variations for the $v=2, J=1-0$ transition. 

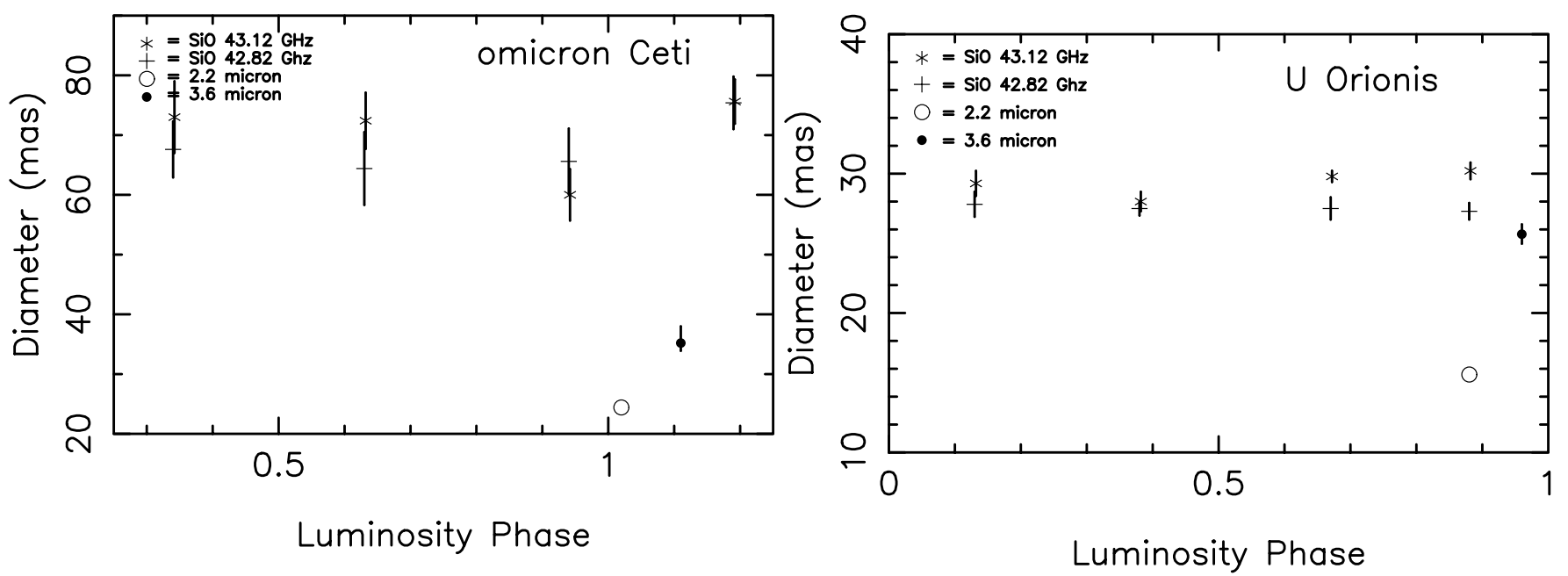

Fig. 11. Left: The $\mathrm{SiO}$ and IR diameters as a function of luminosity phase for omicron Ceti (Mira). Vertical bars on the maser symbols represent the width of the ring as determined from the second moment about the diameter of the ring. The vertical bars on the $3.6 \mu \mathrm{m}$ symbols are error bars. IR diameters are from Mennesson et al. (2002). Right: The SiO and IR diameters as a function of luminosity phase for U Orionis. Note: the $3.6 \mu \mathrm{m}$ diameter is very close to that of the $\mathrm{SiO}$ ring. Vertical bars on the maser symbols represent the width of the ring as determined from the second moment about the diameter of the ring. The vertical bars on the $3.6 \mu \mathrm{m}$ symbols are error bars. IR diameters are from Mennesson et al. (2002).
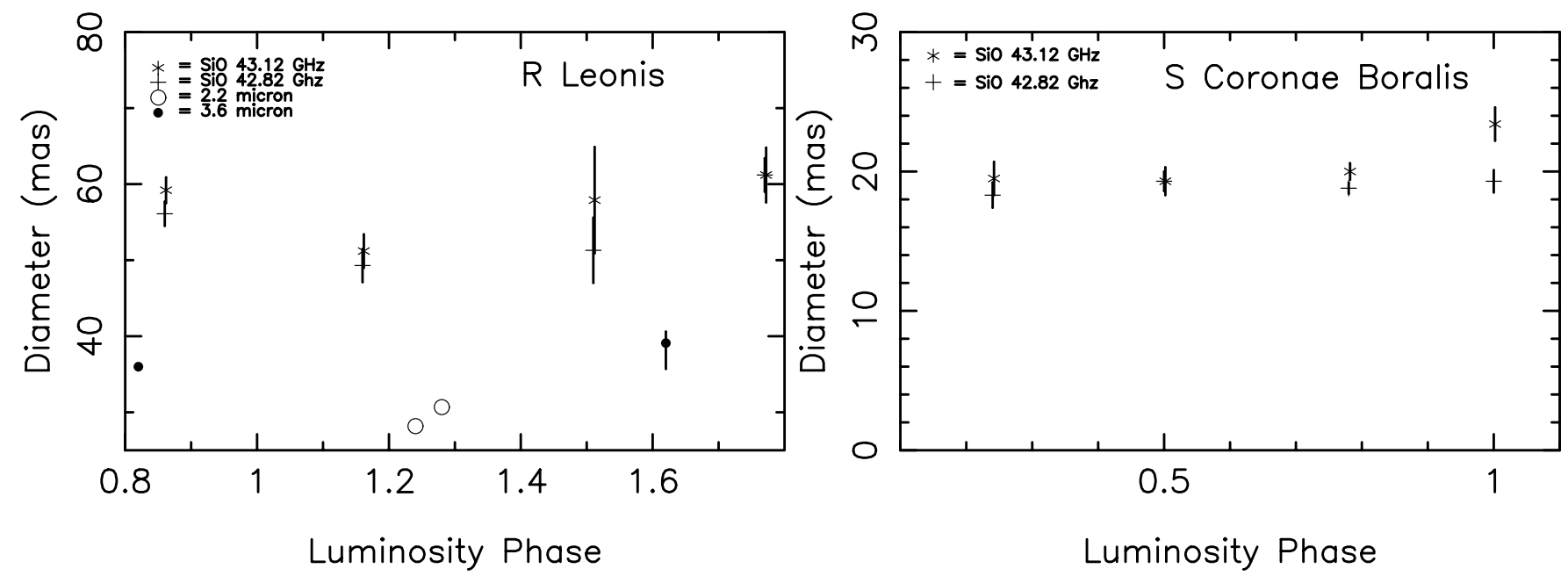

Fig. 12. Left: The SiO and IR diameters as a function of luminosity phase for R Leonis. Right: The SiO diameters as a function of luminosity phase for S Coronae Boralis. IR diameter is from van Belle et al. (2002).

As can be seen, the $\mathrm{SiO}$ ring diameters are generally twice those measured at $2.2 \mu \mathrm{m}$. The six stars for which diameter measurements were available at $2.2,3.6 \mu \mathrm{m}$ and in $\mathrm{SiO}$ are shown in Fig. 16. This figure plots the ratio of the $\mathrm{SiO}$ maser ring diameter to the $3.6 \mu \mathrm{m}$ diameter against the ratio of $3.6 \mu \mathrm{m}$ to $2.2 \mu \mathrm{m}$ diameters. The size of the bars in the plot represent the uncertainty in the determination with a minimum size to assure that each point is visible. There appears to be a significant anticorrelation (correlation coefficient -0.30 ) between the $2.2 / 3.6 \mu \mathrm{m}$ ratio with the $\mathrm{SiO} / 3.6 \mu \mathrm{m}$ ratio, in particular, two stars (U Orionis and R Aquarii) stand out by having nearly the same diameter at $3.6 \mu \mathrm{m}$ as that of the $\mathrm{SiO}$ maser ring while being nearly twice the size at $3.6 \mu \mathrm{m}$ as at $2.2 \mu \mathrm{m}$. This correlation is due to the size observed at $3.6 \mu \mathrm{m}$ since the $2.2 \mu \mathrm{m}$ and $\mathrm{SiO}$ maser ring sizes have a fairly constant ratio.
The cause of this diameter ratio correlation is uncertain but appears to be the result of varying opacity and temperature effects at $3.6 \mu \mathrm{m}$ in the molecular envelope. Since the $3.6 \mu \mathrm{m}$ apparent diameter varies up to the size of the $\mathrm{SiO}$ maser ring, the masers may lie close to the outer boundary of the molecular region. The inner size of the dust shell reported by Danchi et al. (1994) also appears to show a related effect. The two stars with the smallest $\mathrm{SiO}$ ring to $3.6 \mu \mathrm{m}$ diameter ratio have significantly larger inner dust shell radii, $8 R_{2.2} \mu \mathrm{m}$ for R Aquarii and $10 R_{2.2 \mu \mathrm{m}}$ for $\mathrm{U}$ Orionis, suggesting that the dust condenses further from the photosphere in these stars. On the other end of the correlation, omicron Ceti which has the largest $\mathrm{SiO}$ ring to $3.6 \mu \mathrm{m}$ ring diameter ratio has an inner dust ring radius only approximately $5 R_{2.2 \mu \mathrm{m}}$, indicating rapid dust condensation. While the number of cases is small, stars with higher $3.6 \mu \mathrm{m}$ 

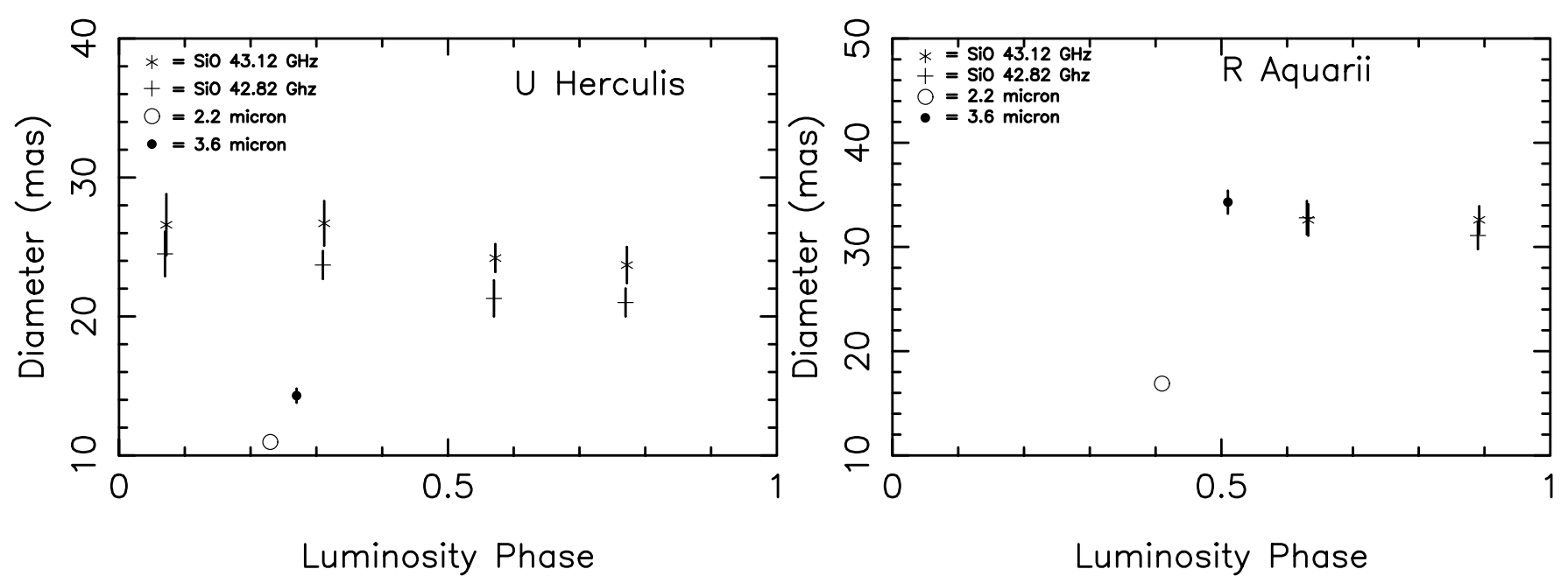

Fig. 13. Left: The SiO and IR diameters as a function of luminosity phase for U Herculis. Vertical bars on the maser symbols represent the width of the ring as determined from the second moment about the diameter of the ring. The vertical bar on the $3.6 \mu \mathrm{m}$ symbol is an error bar. IR diameters are from Mennesson et al. (2002). Right: The SiO and IR diameters as a function of luminosity phase for R Aquarii. Note: the $3.6 \mu \mathrm{m}$ diameter is very close to that of the $\mathrm{SiO}$ ring. IR diameters are from Mennesson et al. (2002).

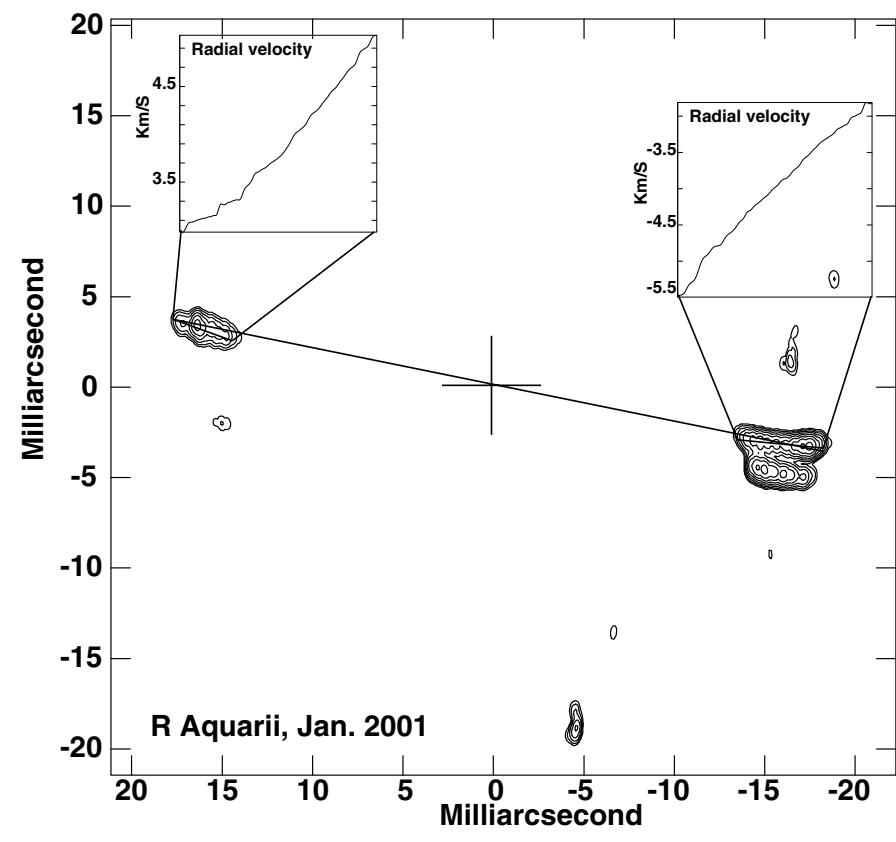

Fig. 14. Total intensity contours of $\mathrm{R}$ Aquarii in the $v=1, J=$ 1-0 transition in January 2001 with inset plots showing the run of radial velocity in slices through the two extended linear features. Velocities are with respect to the systemic velocity of $-24.5 \mathrm{~km} \mathrm{~s}^{-1}$. A cross marks the fitted center of the star and a line connects the extended features on either side.

opacities in the envelope appear to form dust further from the photosphere.

\subsection{Maser ring diameters}

Figures 11-13 indicate that the diameter of the ring in the $v=2, J=1-0$ transition is always smaller and less variable than that in the $v=1, J=1-0$ transition The shock pumped model of Humphreys et al. (1996) predict that the $v=2$,

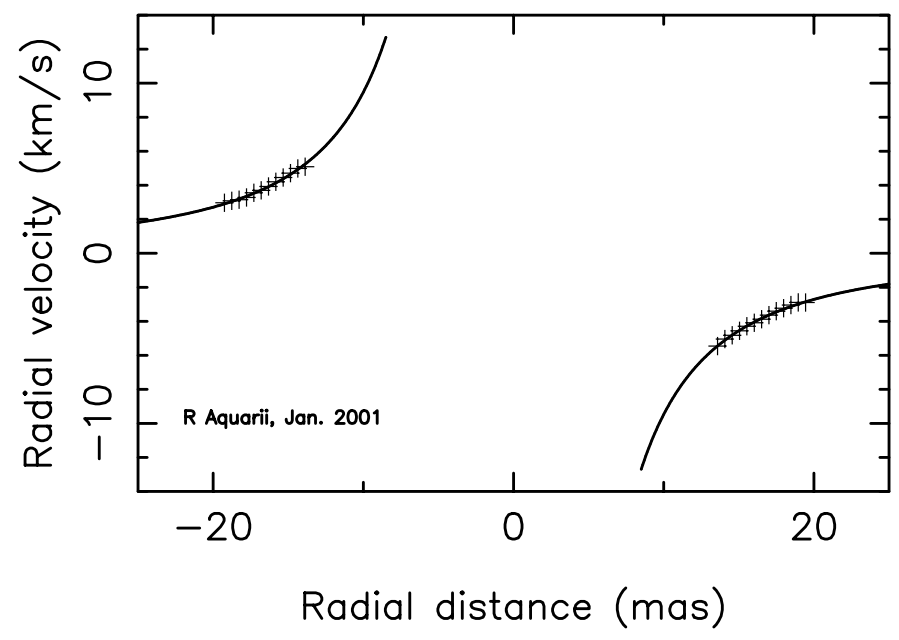

Fig. 15. The velocity data (crosses) along a cut through $\mathrm{R}$ Aquarii in the $v=1, J=1-0$ transition in January 2001 with a model velocity profile (line). The model rotational velocity at the ring radius of 16.3 mas was $3.92 \mathrm{~km} \mathrm{~s}^{-1}$ and scaled with distance from the star as $r^{-1.81}$. The model is shown only from the diameter of the star at $2.2 \mu \mathrm{m}$.

$J=1-0$ transition should produce a smaller diameter ring than the $v=1, J=1-0$ transition. The models of Humphreys et al. (2002) in which variations in the $\mathrm{SiO}$ maser emission is due to shocks predict an rms variation in the measured ring diameter of $6 \%$ over a luminosity cycle. The apparent size of the ring decreases when the shock from a new stellar pulsation pushes new material into the $\mathrm{SiO}$ maser zone. Since the pulsation period of the stars presented here is approximately one year and the span of the observations nearly a year, reasonable, if sparse, sampling was made of a luminosity cycle. The mean and rms variations in the fitted ring diameters for each of the stars observed in all four sessions is given in Table 5 for each of the two transitions observed. 
Table 5. SiO ring diameter variations.

\begin{tabular}{|c|c|c|c|c|c|c|c|c|}
\hline Star & $\begin{array}{r}\text { Mean }^{1} \\
(\mathrm{mas})\end{array}$ & $\begin{array}{r}\text { Mean } \\
\left(2 R_{2.2 \mu \mathrm{m}}\right)\end{array}$ & $\begin{array}{c}\mathrm{rms}^{1} \\
(\mathrm{mas})\end{array}$ & $\begin{array}{r}\text { percent }^{1} \\
(\%)\end{array}$ & $\begin{array}{r}\text { Mean }^{2} \\
\text { (mas) }\end{array}$ & $\begin{array}{r}\text { Mean } \\
\left(2 R_{2.2 \mu \mathrm{m}}\right)\end{array}$ & $\begin{array}{r}\mathrm{rms}^{2} \\
(\%)\end{array}$ & $\overline{\text { percent }^{2}}$ \\
\hline omicron Ceti & 70.3 & 2.88 & 7.0 & 10 & 68.3 & 2.80 & 5.0 & 7 \\
\hline U Orionis & 29.3 & 1.88 & 1.0 & 3 & 27.5 & 1.76 & 0.2 & 0.7 \\
\hline R Leonis & 57.4 & 1.95 & 4.3 & 8 & 54.5 & 1.85 & 5.3 & 10 \\
\hline S Coronae Boralis & 20.6 & 1.82 & 1.9 & 9 & 18.9 & 1.67 & 0.5 & 3 \\
\hline U Herculis & 25.3 & 2.30 & 1.6 & 9 & 22.6 & 2.06 & 1.7 & 8 \\
\hline R Aquarii & 32.6 & 1.93 & & & 31.9 & 1.90 & & \\
\hline R Cassopeiae & 49.9 & 2.01 & & & 45.9 & 1.85 & & \\
\hline
\end{tabular}

${ }^{1} v=1, J=1-0$ transition of $\mathrm{SiO}$ at $43.1 \mathrm{GHz}$.

${ }^{2} v=2, J=1-0$ transition of $\mathrm{SiO}$ at $42.8 \mathrm{GHz}$.

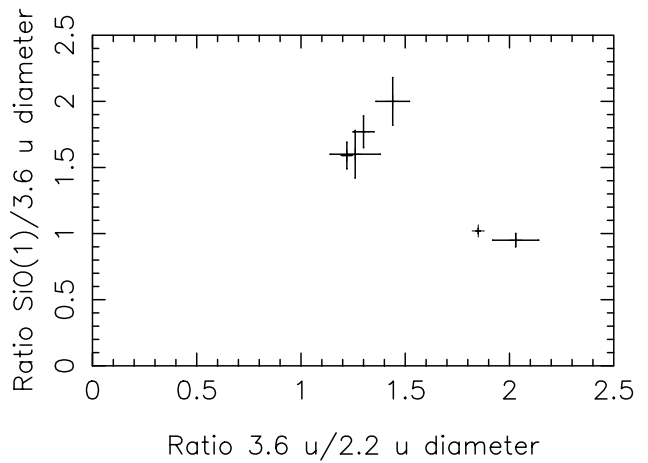

Fig. 16. The ratio of the $\mathrm{SiO}$ maser ring diameter for the $v=1$, $J=1-0$ transition to the $3.6 \mu \mathrm{m}$ diameter against the ratio of the $3.6 \mu \mathrm{m}$ to $2.2 \mu \mathrm{m}$ diameters for o Ceti, U Ori, R Leo, U Her, R Aqr, and $\mathrm{R}$ Cas. The bars shown represent the uncertainties derived from the measurement errors of the $2.2 \mu \mathrm{m}$ and $3.6 \mu \mathrm{m}$ diameters and the width of the $\mathrm{SiO}$ maser ring.

Since the maser rings are frequently sparsely populated with maser spots, the accuracy of the estimation of the diameter of the ring is limited by the number and placements of the spots. All of the stars given in Table 5 show smooth variations in the ring diameter and the two transitions track each other. All are roughly in agreement with the Humphreys et al. (2002) rms variation of $6 \%$ except $U$ Orionis which is nearly constant in size. U Orionis was one of the two stars for which the $3.6 \mu \mathrm{m}$ size is approximately that of the $\mathrm{SiO}$ maser ring. The other is $\mathrm{R}$ Aquarii which was only observed in two sessions so no variation is given in Table 5; however, the two pairs of diameters given in Table 2 for $\mathrm{R}$ Aquarii are quite close. This raises the intriguing possibility that masers in which the ring diameters are the same as that of the molecular material seen at $3.6 \mu \mathrm{m}$ have very stable $\mathrm{SiO}$ maser ring diameters.

The variation of the ring diameter with luminosity phase shown in Figs. 11-13 varies dramatically from star to star. This disagrees with the models of (Humphreys et al. 2002) which predicts that the behavior of the ring diameters with luminosity phase should be similar for all stars. It can also be seen from Table 5 that the $\mathrm{SiO}$ ring diameters cluster around $4 R_{2.2} \mu \mathrm{m}$ and are generally less that the inner diameters of the dust shell reported by Danchi et al. (1994).

\subsection{Rotation of the envelope}

Since the detectable masers are largely in the tangent through the $\mathrm{SiO}$ maser layer, all radial velocities, as seen by us, will generally be azithmutal to the star. Thus, the variation in velocity of maser spots in location in the ring and in time is due either to rotation of the star or turbulent motions in the atmosphere. Table 3 gives the flux density weighted average radial velocity observed in 8 sectors around each maser ring. There is some correlation between the velocities observed in the two transitions, as would be expected if the masers arise in the same regions. There are large variations in the sector average velocities in time, even in sectors with a reasonable flux density of masers. These fluctuations of a few $\mathrm{km} \mathrm{s}^{-1}$ are undoubtedly due to changes in the large scale bulk motions in the stellar atmosphere.

The large bulk non-radial motions seen in the maser spots will tend to mask the signature of the rotation of the star. The stars must be slow rotators due to their enormous size but Fig. 14 strongly suggest rotation is detectable in R Aquarii. Random motions should average out whereas motions due to stellar rotation will not. Figure 17 shows the epoch and transition sector average for the sources in our sample. R Aquarii and $\mathrm{S}$ Coronae Boralis show possible rotation of $3-4 \mathrm{~km} \mathrm{~s}^{-1}$ but the others show no convincing evidence of rotation.

\subsection{Magnetic field geometry}

The theory relating magnetic fields and the polarization of maser emission is described in great detail in Nedoluha \& Watson (1994), Elitzur (1991, 1993, 1996, 1998). Application of the theory to observations is discussed in Reid (1990), Vlemmings et al. (2002) and Kemball \& Diamond (1997) which gives an analysis of the TX Cam results. Kemball \& Diamond (1997) interpret the predominantly tangential polarization vectors seen in TX Cam as evidence of a predominantly radial magnetic field. Unlike TX Cam, there are only a few cases in Figs. 2-10 in which the polarization of the inner portion of the ring is tangential but neither this nor any other prominent pattern is a persistent feature in the polarization of any of the stars observed.

Vlemmings et al. (2002) present circular polarization measurements of $\mathrm{H}_{2} \mathrm{O}$ masers in a number of stars including $\mathrm{U}$ Herculis. The circular polarization is interpreted as Zeeman 

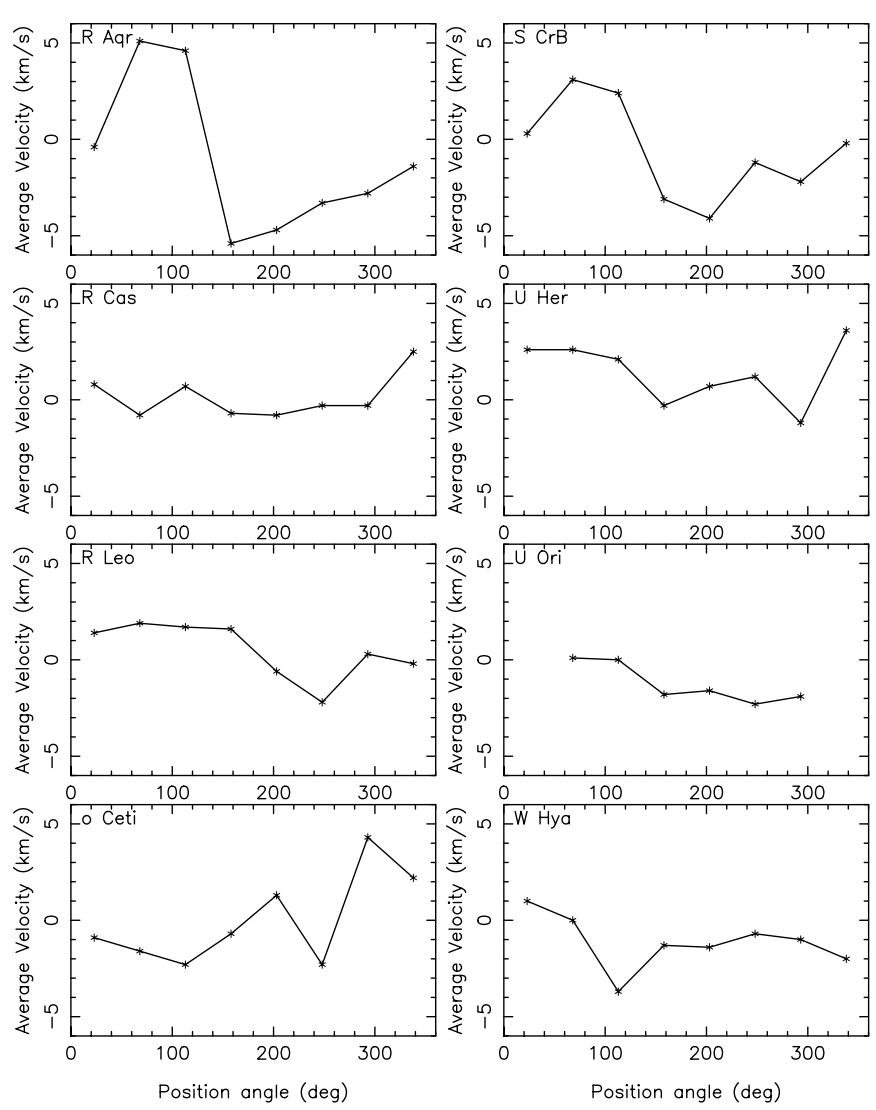

Fig. 17. The sector averaged velocities over epoch and transition.

splitting giving a direct measure of one component of the magnetic field. Their conclusion was that magnetic pressure dominated the thermal in the regions of the $\mathrm{H}_{2} \mathrm{O}$ masers. Analysis of the circular polarization in the data presented here will be given in a future publication.

\section{Conclusions}

We present new total intensity and linear polarization VLBA observations of the $v=2$ and $v=1 J=1-0$ maser transitions of $\mathrm{SiO}$ at 42.8 and $43.1 \mathrm{GHz}$ in a number of Mira stars over a substantial fraction of their pulsation periods. Nine stars were observed at from one to four epochs during 2001. The orientation of the polarization vectors for several sets of observations suggest an ordered magnetic field as claimed by Kemball \& Diamond (1997) in TX Cam; but for most stars at most epochs any such ordering is not apparent. The lifetime of individual spots is generally shorter than the interval between observations (three months) so motions of individual features could not be tracked. The emission is largely confined to a ring about $4 R_{2.2 \mu \mathrm{m}}$ in diameter which is interior to the inner radius of the dust shell reported by Danchi et al. (1994).

Most of the well observed stars show variations in the $\mathrm{SiO}$ ring diameter with a range of rms variations $(5 \%-10 \%)$ consistent with the models of Humphreys et al. (2002). The relationship between ring diameter and luminosity phase was not consistent among different stars; this differs from the model predictions. In addition, U Orionis showed remarkably small variation in its ring diameter at a level inconsistent with the models of (Humphreys et al. 2002). U Orionis was also one of two stars for which the $\mathrm{SiO}$ ring diameter corresponded to the size of the hot molecular layer as measured at $3.6 \mu \mathrm{m}$ (Mennesson et al. 2002). The other such star is R Aquarii, which was only observed in two sessions and little can be said about the variations of ring diameter with epoch except that there was very little difference in the ring diameters over three months.

There appears to be a correlation between the $2.2 / 3.6 \mu \mathrm{m}$ diameter ratio with that of the $\mathrm{SiO} / 3.6 \mu \mathrm{m}$ diameter ratio. Since the $\mathrm{SiO} / 2.2 \mu \mathrm{m}$ diameter ratio is clustered around 2 , this effect is due to the $3.6 \mu \mathrm{m}$ diameters. This is interpreted by Perrin et al. (2003) as opacity differences in a molecular layer. Previous measurements of the inner size of the dust shell (Danchi et al. 1994) suggest that the stars with the smallest $\mathrm{SiO} / 3.6 \mu \mathrm{m}$ ratio also had dust condensation furthest from the photosphere, this may suggest a difference in the temperature structure as well.

The symbiotic star R Aquarii is quite remarkable in another way; in the January 2001 observations, the emission is dominated by regions of emission which are very extended in the radial direction and are very symmetrically located around the center. The velocity field in these regions is interpreted as showing differential rotation in an equatorial disk of material in the star's atmosphere (see Fig. 15). Systematic variations of the measured radial velocity with position angle along the $\mathrm{SiO}$ ring in S Coronae Boralis also suggest a rotation of $3-4 \mathrm{~km} \mathrm{~s}^{-1}$ but the time variable, peculiar velocities make the case less clear than for R Aquarii.

\section{References}

Boboltz, D. A., Diamond, P. J., \& Kemaball, A. J. 1997, ApJ, 487, L147

Bowen, G. H. 1988, ApJ, 329, 299

Cernicharo, J., Brunswig, W., Paubert, G., \& Liechti, S. 1994, ApJ, 423, L143

Colomer, F., Baudry, A., Graham, D. A., et al. 1996, A\&A, 312, 950

Colomer, F., Graham, D. A., Krichbaum, T. P., et al. 1992, A\&A, 254, L17

Cotton, W. D. 1993, AJ, 106, 1241

Coude Du Foresto, V., Perrin, G., Ruilier, C., et al. 1998, in Astronomical Interferometry, ed. R. D. Reasenberg, Proc. SPIE, 3350,856

Danchi, W. C., Bester, M., Degiacomi, C. G., Greenhill, L. J., \& Townes, C. H. 1994, AJ, 107, 1469

Diamond, P. J., \& Kemball, A. J. 1998, in ASP Conf. Ser. 144, Radio Emission from Galactic and Extragalactic Compact Sources, IAU Colloq, 164, 245

Diamond, P. J., \& Kemball, A. J. 1999, in Asymptotic Giant Branch Stars, IAU Symp., 191, 195

Diamond, P. J., Kemball, A. J., Junor, W., et al. 1994, ApJ, 430, L61

Elitzur, M. 1991, ApJ, 370, 407

Elitzur, M. 1993, ApJ, 416, 256

Elitzur, M. 1996, ApJ, 457, 415

Elitzur, M. 1998, ApJ, 504, 390

Engels, D., \& Heske, A. 1989, A\&AS, 81, 323

ESA 1997, VizieR Online Data Catalog, 1239, 0

Gray, M. D., Ivison, R. J., Humphreys, E. M. L., \& Yates, J. A. 1998, MNRAS, 295, 970 
Hinkle, K. H., Hall, D. N. B., \& Ridgway, S. T. 1982, ApJ, 252, 697

Hollis, J. M., Bertram, R., Wagner, R. M., \& Lampland, C. O. 1999, ApJ, 514, 895

Hollis, J. M., Boboltz, D. A., Pedelty, J. A., White, S. M., \& Forster, J. R. 2001, ApJ, 559, L37

Humphreys, E. M. L., Gray, M. D., Yates, J. A., et al. 1996, MNRAS, 282,1359

Humphreys, E. M. L., Gray, M. D., Yates, J. A., et al. 2002, A\&A, 386, 256

Kemball, A. J., \& Diamond, P. J. 1997, ApJ, 481, L111

Lane, A. P., Ho, P. T. P., Predmore, C. R., et al. 1980, in Interstellar Molecules, IAU Symp., 87, 535

McIntosh, G. C., Predmore, C. R., Moran, J. M., et al. 1989, ApJ, 337, 934

Mennesson, B., Mariotti, J. M., Coudé Du Foresto, V., et al. 1999, A\&A, 346, 181

Mennesson, B., Perrin, G., Chagnon, G., et al. 2002, ApJ, 579, 446

Moran, J. M., Ball, J. A., Predmore, C. R., et al. 1979, ApJ, 231, L67

Nedoluha, G. E., \& Watson, W. D. 1994, ApJ, 423, 394

Perrin, G., Coudé du Foresto, V., Ridgway, S. T., et al. 1999, A\&A, 345,221
Perrin, G., et al. 2003, A\&A, in preparation

Phillips, R. B., Sivakoff, G. R., Lonsdale, C. J., \& Doeleman, S. S. 2001, AJ, 122, 2679

Phillips, R. B., Straughn, A. H., Doeleman, S. S., \& Lonsdale, C. J. 2003, ApJ, 588, L105

Reid, M. J. 1990, Galactic and Intergalactic Magnetic Fields, in IAU Symp., 140, 21

Reid, M. J., \& Menten, K. M. 1997, ApJ, 476, 327

Reid, M. J., \& Moran, J. M. 1981, ARA\&A, 19, 231

Traub, W. A., Carleton, N. P., Bregman, J. D., et al. 2000, in Interferometry in Optical Astronomy, ed. P. J. Lena, \& A. Quirrenbach, Proc. SPIE, 4006, 715

van Belle, G. T., Thompson, R. R., \& Creech-Eakman, M. J. 2002, AJ, 124, 1706

Vlemmings, W. H. T., Diamond, P. J., \& van Langevelde, H. J. 2002, A\&A, 394, 589

Wittkowski, M., \& Bobolz, D. 2003, in Future Directions in High Resolution Astronomy: The 10th Anniversary of the VLBA, ed. J. D. Romney, \& M. J. Reid, to appear in ASP Conf. Ser. 